\title{
Existence and Controllability Results for Nonlocal Fractional Impulsive Differential Inclusions in Banach Spaces
}

\author{
JinRong Wang ${ }^{1,2}$ and Ahmed G. Ibrahim ${ }^{3}$ \\ ${ }^{1}$ School of Mathematics and Computer Science, Guizhou Normal College, Guiyang, Guizhou 550018, China \\ ${ }^{2}$ Department of Mathematics, Guizhou University, Guiyang, Guizhou 550025, China \\ ${ }^{3}$ Department of Mathematical, Faculty of Science, King Faisal University, Al-Ahsa 31982, Saudi Arabia
}

Correspondence should be addressed to JinRong Wang; wjr9668@126.com

Received 24 May 2013; Accepted 5 August 2013

Academic Editor: Henryk Hudzik

Copyright (C) 2013 J. Wang and A. G. Ibrahim. This is an open access article distributed under the Creative Commons Attribution License, which permits unrestricted use, distribution, and reproduction in any medium, provided the original work is properly cited.

We firstly deal with the existence of mild solutions for nonlocal fractional impulsive semilinear differential inclusions involving Caputo derivative in Banach spaces in the case when the linear part is the infinitesimal generator of a semigroup not necessarily compact. Meanwhile, we prove the compactness property of the set of solutions. Secondly, we establish two cases of sufficient conditions for the controllability of the considered control problems.

\section{Introduction}

During the past two decades, fractional differential equations and inclusions have gained considerable importance due to their applications in various fields, such as physics, mechanics, and engineering. For some of these applications, one can see [1-3] and the references therein. El-Sayed and Ibrahim [4] initiated the study of fractional multivalued differential inclusions. Recently, some basic theory for initial value problems for fractional differential equations and inclusions was discussed in [5-13].

The theory of impulsive differential equations and inclusions has been an object of interest because of its wide applications in physics, biology, engineering, medical fields, industry, and technology. The reason for this applicability arises from the fact that impulsive differential problems are an appropriate model for describing process which at certain moments change their state rapidly and which cannot be described using the classical differential problems. During the last ten years, impulsive differential inclusions with different conditions have been intensely studented by many mathematicians. At present, the foundations of the general theory of impulsive differential equations and inclusions are already laid, and many of them are investigated in details in the book of Benchohra et al. [14].
Moreover, a strong motivation for investigating the nonlocal Cauchy problems, which is a generalization for the classical Cauchy problems with initial condition, comes from physical problems. For example, it used to determine the unknown physical parameters in some inverse heat condition problems. The nonlocal condition can be applied in physics with better effect than the classical initial condition $x(0)=x_{0}$. For example, $g(x)$ may be given by $g(x)=\sum_{k=1}^{k=m} c_{k} x\left(t_{k}\right)$, where $c_{k}(k=1,2, \ldots, m)$ are given constants and $0<$ $t_{1}<t_{2}<\cdots<t_{m}<b$. In the few past years, several papers have been devoted to study the existence of solutions for differential equations or inclusions with nonlocal conditions [15-17]. For impulsive differential equations or inclusions with nonlocal conditions of order we refer to $[16,17]$. For impulsive differential equations or inclusions of fractional order we refer to $[9,18-21]$ and the references therein.

Motivated by the researches mentioned previously, we will study the following nonlocal impulsive differential inclusions of fractional order $\alpha \in(0,1)$ of the type:

$$
\begin{gathered}
{ }^{c} D^{\alpha} x(t) \in A x(t)+F(t, x(t)), \quad \text { a.e. on } \\
J-\left\{t_{1}, t_{2}, \ldots, t_{m}\right\}, \quad J=[0, b], b>0,
\end{gathered}
$$




$$
\begin{gathered}
x(0)=x_{0}-g(x), \\
x\left(t_{k}^{+}\right)=x\left(t_{k}\right)+I_{k}\left(x\left(t_{k}\right)\right), \quad k=1,2, \ldots, m,
\end{gathered}
$$

where ${ }^{c} D^{\alpha}$ is the Caputo derivative of order $\alpha$ with the lower limit zero, $A: D(A) \subseteq E \rightarrow E$ is the infinitesimal generator of a $C_{0}$-semigroup $\{T(t), t \geq 0\}$ on a real Banach space $E$ which in not necessarily compact, $F: J \times E \rightarrow 2^{E}$ is a multifunction, $0=t_{0}<t_{1}<\cdots<t_{m}<t_{m+1}=b$, $I_{k}: E \rightarrow E(k=1,2, \ldots, m)$ impulsive functions which characterize the jump of the solutions at impulsive points, $g: P C(J, E) \rightarrow E$ is a nonlinear function related to the nonlocal condition at the origin, and $x\left(t_{k}^{+}\right), x\left(t_{k}^{-}\right)$are the right and left limits of $x$ at the point $t_{k}$, respectively.

To study the theory of abstract impulsive differential inclusions with fractional order, the first step is how to define the mild solution. Mophou [18] firstly introduced a concept on a mild solution which was inspired by Jaradat et al. [19]. However, it does not incorporate the memory effects involved in fractional calculus and impulsive conditions. Wang et al. [9] introduced a new concept of PC-mild solutions for (1) and derived existence and uniqueness results concerning the $P C$-mild solutions for (1) when $F$ is a Lipschitz single-valued function or continuous and maps bounded sets into bounded sets, and $T(t), t>0$, is compact.

In order to do a comparison between our obtained results in this paper and the known recent results in the same domain, we refer to the following: Ouahab [8] proved a version of Filippov's Theorem for (1) without impulse and $A$ is an almost sectorial operator, Cardinali and Rubbioni [16] proved the existence of mild solutions to (1) when $\alpha=1$ and the multivalued function $F$ satisfies the lower ScorzaDragoni property, and $\{A(t)\}_{t \geq 0}$ is a family of linear operator, generating a strongly continuous evolution operators, Fan [17] studied a nonlocal Cauchy problem in the presence of impulses, governed by autonomous semilinear differential equation, Dads et al. [20] and Henderson and Ouahab [21] considered (1) when $A=0$, and Zhou and Jiao $[12,13]$ introduced a suitable definition of mild solution for (1) based on Laplace transformation and probability density functions for (1) when $F$ is single-valued function and without impulse. More recently, Wang and Zhou [10] proved existence and controllability results for (1) without impulse and with local conditions. Very recently, Zhang and Liu [11] considered (1) without impulse, $F$ is a single-valued function, and $\{T(t), t>$ $0\}$ is strongly equicontinuous $C_{0}$-semigroup. Ibrahim and Al Sarori [22] gave some existence results of mild solutions for nonlocal impulsive differential inclusions with delay and of fractional order in Caputo sense when the semigroup is compact. Among the previous works, little is concerned with nonlocal impulsive fractional differential inclusions via noncompact semigroup and the techniques of the measure of noncompactness.

In Section 3, we apply the methods and techniques to derive some sufficient conditions for existence results for (1) when the values of the multivalued function $F$ are nonempty convex and compact. At the end of this section, we prove that the set of mild solutions of (1) is compact. We adopt the definition of mild solution introduced by Wang et al. [9]. Unlike the papers $[9,10,12,13,22]$, we do not assume $\{T(t), t \geq 0\}$ is a compact semigroup, and instead we assume that $F$ satisfies a compactness condition involving the Hausdorff measure of noncompactness.

The following are some simple examples for operators that generate a noncompact semigroup.

(i) The ordinary differential operator $A$ on the normed space $X=L^{2}(-\infty, \infty)$ with domain $D\left(A_{1}\right)=$ $H^{1}(-\infty, \infty)$ generates a noncompact semigroup $T_{1}(t), t \geq 0$, defined by

$$
\begin{array}{ll}
\left(T_{1}(t) u\right)(s)=u(t+s), & \text { for any } u \in X \\
& \text { and any } t, s \in(-\infty, \infty) .
\end{array}
$$

(ii) The ordinary differential operator $A$ on the normed space $X=C(0, \infty)$ with domain $D\left(A_{1}\right)=C^{1}(0, \infty)$ generates a noncompact semigroup $T_{2}(t), t \geq 0$, defined by

$$
\begin{array}{ll}
\left(T_{2}(t) u\right)(s)=u(t+s), & \text { for any } u \in X \\
& \text { and any } t, s \in(0, \infty) .
\end{array}
$$

In Section 4, we will consider the following two nonlocal semilinear impulsive evolution system of order $\alpha$ of the type

$$
\begin{array}{r}
{ }^{c} D^{\alpha} x(t) \in A x(t)+F(t, x(t))+B(u(s)), \\
\text { a.e. on } J-\left\{t_{1}, t_{2}, \ldots, t_{m}\right\}, \\
x(0)=x_{0}-g(x), \\
x\left(t_{k}^{+}\right)=x\left(t_{k}\right)+I_{k}\left(x\left(t_{k}^{-}\right)\right), \quad k=1,2, \ldots, m, \\
{ }^{c} D^{\alpha} x(t) \in A x(t)+F(t, x(t))+(V u)(s), \\
\text { a.e. on } J-\left\{t_{1}, t_{2}, \ldots, t_{m}\right\}, \\
x(0)=x_{0}-g(x), \\
x\left(t_{k}^{+}\right)=x\left(t_{k}\right)+I_{k}\left(x\left(t_{k}^{-}\right)\right), \quad k=1,2, \ldots, m,
\end{array}
$$

where the control function $u$ is given in $L^{2}(J, X)$, a Banach space of admissible control functions with $X$ being a Banach space, and $B$ is a bounded linear operator from $X$ into $E$ and $V: L^{2}(J, X) \rightarrow L^{1 / \sigma}(J, E), \sigma \in(0, \alpha)$.

Most previous controllability works contained the assumption of the compactness of the operator semigroup. However, Hernández and O’Regan [23] and Obukhovski and Zecca [24] pointed that if the operator semigroup was compact, then the assumption (HW) (see Section 4) was valid if and only if $E$ was finite dimensional. Controllability of (4) when $\alpha=1$ was discussed by Guo et al. [25]. We refer to, in recent years, the problem of controllability for various kinds of fractional differential and integro-differential equations and inclusions have been discussed in [26-28].

The present paper is organized as follows. In Section 2, we collect some background material and basic results from 
multivalued analysis and fractional calculus to be used later. In Section 3, we prove existence results for (1); we also show that the set of solutions is compact. In Section 4, we present two interesting controllability results of (4) and (5).

Our basic tools are the methods and results for semilinear differential inclusions, the properties of noncompact measure, compactness criterion in the piecewise continuous functions of space, and fixed point techniques.

\section{Preliminaries and Notation}

Let $C(J, E)$ the space of $E$-valued continuous functions on $J$ with the uniform norm $\|x\|=\sup \{\|x(t)\|, t \in J\}, L^{1}(J, E)$ the space of $E$-valued Bochner integrable functions on $J$ with the norm $\|f\|_{L^{1}(J, E)}=\int_{0}^{b}\|f(t)\| d t, P_{b}(E)=\{B \subseteq E: B$ is nonempty and bounded $\}, P_{\mathrm{cl}}(E)=\{B \subseteq E: B$ is nonempty and closed $\}, P_{k}(E)=\{B \subseteq E: B$ is nonempty and compact $\}$, $P_{\mathrm{cl}, \mathrm{cv}}(E)=\{B \subseteq E: B$ is nonempty, closed, and convex $\}$, and $P_{\mathrm{ck}}(E)=\{B \subseteq E: B$ is nonempty, convex, and compact $\}$, and $\operatorname{conv}(B)$ (resp., $\overline{\operatorname{conv}}(B))$ be the convex hull (resp., convex closed hull in $E$ ) of a subset $B$.

Definition 1. Let $X$ and $Y$ be two topological spaces. A multifunction $G: X \rightarrow P(Y)$ is said to be upper semicontinuous (u.s.c.) if $G^{-1}(V)=\{x \in X: G(x) \subseteq V\}$ is an open subset of $X$ for every open $V \subseteq Y$. $G$ is called closed if its graph $\Gamma_{G}=\{(x, y) \in X \times Y: y \in G(x)\}$ is closed subset of the topological space $X \times Y$. $G$ is said to be completely continuous if $G(B)$ is relatively compact for every bounded subset $B$ of $X$. If the multifunction $G$ is completely continuous with nonempty compact values, then $G$ is u.s.c. if and only if $G$ is closed.

Lemma 2 (see [29, Theorem 8.2.8]). Let $(\Omega, A, \mu)$ be a complete $\sigma$-finite measure space, $X$ a complete separable metric space, and $F: \Omega \rightarrow 2^{X}$ a measurable multivalued function with nonempty closed images. Consider a multivalued function $G$ from: $\Omega \times X$ to $P(Y) ; Y$ is a complete separable metric space such that for every $x \in X$ the multivalued function $w \rightarrow$ $G(w, x)$ is measurable, and for every $w \in \Omega$ the multivalued function $x \rightarrow G(w, x)$ is continuous. Then the multivalued function $w \rightarrow \overline{G(w, F(w))}$ is measurable. In particular for every measurable single-valued function $z: \Omega \rightarrow X$, the multivalued function $w \rightarrow G(w, z(w))$ is measurable, and for every Caratheodory single-valued function $\varphi: \Omega \times X \rightarrow Y$, the multivalued function $w \rightarrow \overline{\varphi(w, F(w))}$ is measurable.

Definition 3. A sequence $\left\{f_{n}: n \in \mathbb{N}\right\} \subset L^{1}(J, E)$ is said to be semicompact if

(i) it is integrally bounded; that is, there is $q \in L^{1}\left(J, \mathbb{R}^{+}\right)$ such that

$$
\left\|f_{n}(t)\right\| \leq q(t) \quad \text { a.e. } t \in J
$$

(ii) the set $\left\{f_{n}(t): n \in \mathbb{N}\right\}$ is relatively compact in $E$ a.e., $t \in J$.

We recall one fundamental result which follows from Dunford-Pettis Theorem.
Lemma 4 (see [30]). Every semi-compact sequence in $L^{1}(J, E)$ is weakly compact in $L^{1}(J, E)$.

For more about multifunctions, we refer to [29, 31-34].

Let $(\mathscr{A}, \geq)$ be a partially ordered set. A function $\chi$ : $P_{b}(E) \rightarrow \mathscr{A}$ is called a measure of noncompactness (MNC) in $E$ if $\chi(\overline{\operatorname{conv}} B)=\chi(B)$, for every $B \in P_{b}(E)$.

Definition 5 (see [35]). A measure of noncompactness $\beta$ is called

(i) monotone if $B_{0}, B_{1} \in P_{b}(E), B_{0} \subset B_{1}$ implies $\chi\left(B_{0}\right) \leq$ $\chi\left(B_{1}\right)$;

(ii) nonsingular if $\chi(\{a\} \cup B)=\chi(B)$, for every $a \in E$, $B \in P_{b}(E)$;

(iii) regular if $\chi(B)=0$ is equivalent to the relative compactness of $B$;

(iv) invariant with respect to union with compact sets if for any compact subset $K$ in $E$ and any $B \in P_{b}(E)$, $\chi(B \cup K)=\chi(B) ;$

(v) algebraic semiadditive if $\chi\left(B_{1}+B_{2}\right) \leq \chi\left(B_{1}\right)+\chi\left(B_{2}\right)$, for every $B_{1}, B_{2} \in P_{b}(E)$, where $B_{1}+B_{2}=\{a+b: a \in$ $\left.B_{1}, b \in B_{2}\right\}$

(vi) semiadditive if $\chi\left(B_{1} \cup B_{2}\right)=\max \left\{\chi\left(B_{1}\right), \chi\left(B_{2}\right)\right\}$, for every $B_{1}, B_{2} \in P_{b}(E)$; the Hausdorff measure of noncompactness which is defined as

$\chi(B)=\inf \{\epsilon>0: B$ can be covered by finitely

$$
\text { many balls of radius } \leq \epsilon\} \text {, }
$$

possesses all the properties (i)-(vi) and the following additive properties:

(vii) the Lipschitz property $\left|\chi\left(B_{1}\right)-\chi\left(B_{2}\right)\right| \leq h\left(B_{1}, B_{2}\right)$, for every $B_{1}, B_{2} \in P_{b}(E)$, where $h$ is the Hausdorff distance;

(viii) $\chi(t B)=|t| \chi(B)$, for every $t \in \mathbb{R}, B \in P_{b}(E)$;

(ix) let $L: E \rightarrow E$ be a bounded linear operator. Then $\chi(L(B)) \leq\|L\| \chi(B)$, for every $B \in P_{b}(E)$.

Note that the property (vii) implies the continuity property of $\chi$ with respect to the Hausdorff metric. For more information about the measure of noncompactness, we refer to $[29,34-$ 36].

Lemma 6 (see [29, Lemma 1.1.9]). Let $\left(Z_{n}\right)_{n \geq 1} \subset Z \subset X$ be a sequence of subsets where $Z$ is a compact in the separable Banach space $X$. Then

$$
\overline{\mathrm{co}}\left(\lim \sup _{n \rightarrow \infty} Z_{n}\right)=\bigcap_{N>0} \overline{\mathrm{co}}\left(\bigcup_{n \geq N} Z_{n}\right) .
$$

Lemma 7 (see [31], generalized Cantor's intersection). If $\left(B_{n}\right)_{n \geq 1}$ is a decreasing sequence of nonempty, bounded, and closed subsets of $E$ and $\lim _{n \rightarrow \infty} \chi\left(B_{n}\right)=0$, then $\bigcap_{n=1}^{\infty} B_{n}$ is nonempty and compact. 
Lemma 8 (see [35]). Let $\chi_{C(J, E)}$ be the Hausdorff measure of noncompactness on $C(J, E)$. If $W \subseteq C(J, E)$ is bounded, then for every $t \in J$,

$$
\chi(W(t)) \leq \chi_{C(J, E)}(W),
$$

where $W(t)=\{x(t): x \in W\}$. Furthermore, if $W$ is equicontinuous on $J$, then the map $t \rightarrow \chi\{x(t): x \in W\}$ is continuous on $J$ and $\chi_{C(J, E)}(W)=\sup _{t \in J} \chi\{x(t): x \in W\}$.

Lemma 9 (see [36, Page 125]). Let $B$ be a bounded set in E. Then for every $\varepsilon>0$, there is a sequence $\left(x_{n}\right)_{n \geq 1}$ in B such that

$$
\chi(B) \leq 2 \chi\left\{x_{n}: n \geq 1\right\}+\varepsilon .
$$

Lemma 10 (see [37, Lemma 4]). Let $\left\{f_{n}: n \in \mathbb{N}\right\} \subset L^{P}(J, E)$, $P \geq 1$ be an integrably bounded sequence such that

$$
\chi\left\{f_{n}: n \geq 1\right\} \leq \gamma(t), \quad \text { a.e. } t \in J,
$$

where $\gamma \in L^{1}\left(J, \mathbb{R}^{+}\right)$. Then for each $\epsilon>0$, there exists a compact $K_{\epsilon} \subseteq E$, a measurable set $J_{\epsilon} \subset J$, with measure less than $\epsilon$, and a sequence of functions $\left\{g_{n}^{\epsilon}\right\} \subset L^{P}(J, E)$ such that $\left\{g_{n}^{\epsilon}(t): n \geq 1\right\} \subseteq K_{\epsilon}$, for all $t \in J$ and

$$
\begin{aligned}
\left\|f_{n}(t)-g_{n}^{\epsilon}(t)\right\|<2 \gamma(t)+\epsilon, & \text { for every } n \geq 1 \\
& \text { and every } t \in J-J_{\epsilon} .
\end{aligned}
$$

Lemma 11 (see [10, Lemma 2.10]). For $\tau \in(0,1]$ and $0<e \leq$ $c$, one has $\left|e^{\tau}-c^{\tau}\right| \leq(c-e)^{\tau}$.

Definition 12. The fractional integral of order $\alpha \in(0,1)$ of a function $f \in L^{1}(J, E)$ is defined by

$$
I^{\alpha} f(t)=\int_{0}^{t} \frac{(t-s)^{\alpha-1}}{\Gamma(\alpha)} f(s) d s, \quad t>0,
$$

provided the right side is defined on $J$, where $\Gamma$ is the Euler gamma function defined by $\Gamma(\alpha)=\int_{0}^{\infty} t^{\alpha-1} e^{-t} d t$.

Definition 13. The Caputo derivative of order $\alpha \in(0,1)$ of a continuously differentiable function $f: J \rightarrow E$ is defined by

$$
{ }^{c} D^{\alpha} f(t)=\frac{1}{\Gamma(1-\alpha)} \int_{0}^{t}(t-s)^{-\alpha} f^{(1)}(s) d s=I^{(1-\alpha)} f^{(1)}(t) \text {. }
$$

Note that the integrals appearing in the two previous definitions are taken in Bochner' sense and ${ }^{c} D^{\alpha} I^{\alpha} f(t)=f(t)$ for all $t \in J$. For more information about the fractional calculus we refer to $[2,3]$.

Definition 14 (see [13, Lemma 3.1, and Definition 3.1]; see also [10-12]). Let $h: J \rightarrow E$. A function $x \in C(J, E)$ is said to be a mild solution of the following system:

$$
\begin{gathered}
{ }^{c} D^{\alpha} x(t)=A x(t)+h(t), \quad t \in J, \\
x(0)=x_{0} \in E,
\end{gathered}
$$

if it satisfies the following integral equation:

$$
x(t)=K_{1}(t)\left(x_{0}\right)+\int_{0}^{t}(t-s)^{\alpha-1} K_{2}(t-s) h(s) d s, \quad t \in J,
$$

where

$$
\begin{gathered}
K_{1}(t)=\int_{0}^{\infty} \xi_{\alpha}(\theta) T\left(t^{\alpha} \theta\right) d \theta, \\
K_{2}(t)=\alpha \int_{0}^{\infty} \theta \xi_{\alpha}(\theta) T\left(t^{\alpha} \theta\right) d \theta, \\
\xi_{\alpha}(\theta)=\frac{1}{\alpha} \theta^{-1-(1 / \alpha)} w_{\alpha}\left(\theta^{-(1 / \alpha)}\right) \geq 0,
\end{gathered}
$$

$w_{\alpha}(\theta)=(1 / \pi) \sum_{n=1}^{\infty}(-1)^{n-1} \theta^{-\alpha n-1}(\Gamma(n \alpha+1) / n !) \sin (n \pi \alpha), \theta \epsilon$ $(0, \infty)$ and $\xi_{\alpha}$ are a probability density function defined on $(0, \infty)$; that is, $\int_{0}^{\infty} \xi_{\alpha}(\theta) d \theta=1$.

In the following we recall the properties of $K_{1}(\cdot), K_{2}(\cdot)$.

Lemma 15 (see [13, Lemma 3.2, Lemma 3.3, and Lemma 3.5]).

(i) For any fixed $t \geq 0, K_{1}(t), K_{2}(t)$ are linear bounded operators.

(ii) For $\gamma \in[0,1], \int_{0}^{\infty} \theta^{\gamma} \xi_{\alpha}(\theta) d \theta=\Gamma(1+\gamma) / \Gamma(1+\alpha \gamma)$.

(iii) If $\|T(t)\| \leq M$ for all $t \geq 0$, then for any $x \in E$, $\left\|K_{1}(t) x\right\| \leq M\|x\|$ and $\left\|K_{2}(t) x\right\| \leq(M / \Gamma(\alpha))\|x\|$ for all $t \geq 0$.

(iv) For any fixed $t \geq 0, K_{1}(t), K_{2}(t)$ are strongly continuous.

(v) If $T(t), t>0$ is compact, then $K_{1}(t)$ and $K_{2}(t)$ are compacts.

Let $J_{0}=\left[0, t_{1}\right]$ and $J_{k}=\left(t_{k}, t_{k+1}\right], k=1,2, \ldots, m$. To give the concept of mild solution of (1), we consider the set of functions:

$$
\begin{aligned}
P C & (J, E) \\
= & \left\{x: J \longrightarrow E: x_{\mid J_{i}} \in C\left(J_{i}, E\right), i=0,1,2, \ldots, m,\right. \\
& \left.\quad x\left(t_{i}^{+}\right), x\left(t_{i}^{-}\right) \text {exist for each } i=1,2, \ldots, m\right\} .
\end{aligned}
$$

It is easy to check that $P C(J, E)$ is a Banach space endowed with the norm:

$$
\|x\|_{P C(J, E)}=\max \{\|x(t)\|: t \in J\} .
$$

As in [16] we consider the map $\chi_{P C}: P_{b}(P C(J, E)) \rightarrow$ $[0, \infty)$ defined by

$$
\chi_{P C}(B)=\max _{i=0,1,2, \ldots, m} \chi_{i}\left(B_{\mid \bar{J}_{i}}\right),
$$

where $\chi_{i}$ is the Hausdorff measure of noncompactness on the Banach space $C\left(\bar{J}_{i}, E\right)$ and

$$
\begin{aligned}
B_{\mid \overline{J_{i}}}=\left\{x^{*}: \overline{J_{i}}\right. & \longrightarrow E: x^{*}(t)=x(t), t \in J_{i}, \\
x^{*}\left(t_{i}\right) & \left.=x\left(t_{i}^{+}\right), x \in B\right\}, \quad i=0,1, \ldots, m .
\end{aligned}
$$


Of course $B_{\mid \overline{J_{0}}}=\left\{x_{\mid \overline{J_{0}}}: x \in B\right\}$. It is easily seen that $\chi_{P C}$ is the Hausdorff measure of noncompactness on the Banach space $P C(J, E)$.

By using the concept of mild solutions of impulsive fractional evolution equation in Ouahab [8], we can give the concept of mild solution for our considered problem (1).

Definition 16. By a mild solution for (1), we mean a function $x \in P C(J, E)$ which satisfies the following integral equation:

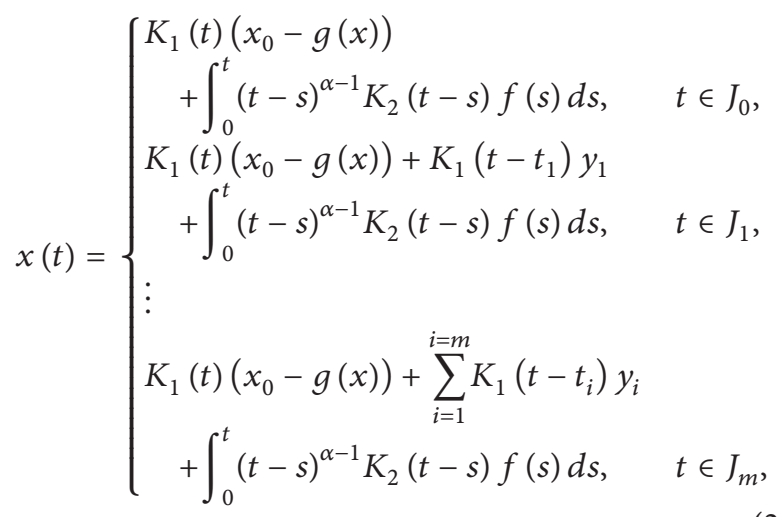

where $y_{i}=I_{i}\left(x\left(t_{i}^{-}\right)\right), i=1, \ldots, m$, and $f$ is an integrable selection for $F(\cdot, x(\cdot))$.

Remark 17. The above definition of piecewise continuous mild solutions comes from Ouahab [8] which is more suitable than the corresponding definition of piecewise continuous mild solutions for impulsive semilinear fractional evolution equations in Shu et al. [38]. In fact, if $\alpha=1$, then (22) reduces to

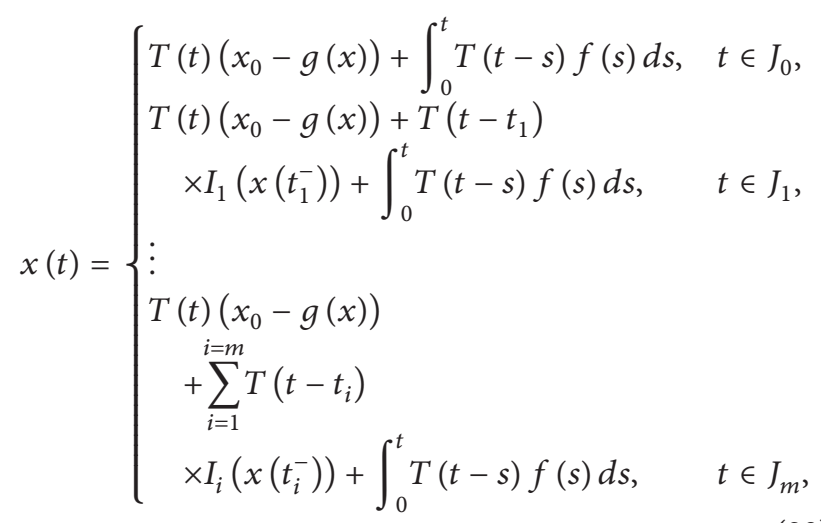

which is the standard formula of PC-mild solutions of impulsive differential inclusions:

$$
\begin{gathered}
x^{\prime}(t) \in A x(t)+F(t, x(t)), \quad \text { a.e. on } J-\left\{t_{1}, t_{2}, \ldots, t_{m}\right\}, \\
x(0)=x_{0}-g(x), \\
x\left(t_{i}^{+}\right)=x\left(t_{i}\right)+I_{i}\left(x\left(t_{i}\right)\right), \quad i=1,2, \ldots, m .
\end{gathered}
$$

However, one cannot expect to obtain the above coincide formula by using the definition of mild solutions in Shu et al.
[38]. For more discussion on the formula of solutions to impulsive fractional differential equations, the reader can refer to Fěckan et al. [39].

Theorem 18 (see [31, Corollary 3.3.1, and Proposition 3.5.1]). If $W$ is a closed convex subset of a Banach space $X$ and $R: W \rightarrow P_{c k}(X)$ is closed and $\gamma$-condensing, where $\gamma$ is a nonsingular measure of noncompactness; then $R$ has a fixed point. If the set of fixed points for $R$ is a bounded subset of $E$, then it is compact.

The following fixed point theorem for contraction multivalued is proved by Covitz and Nadler [30].

Theorem 19. Let $(X, d)$ be a complete metric space. If $R: X \rightarrow$ $P_{c l}(X)$ is contraction, then $R$ has a fixed point.

\section{Existence of Mild Solutions for the Problem (1)}

Theorem 20. Let $A$ be the infinitesimal generator of a $C_{0^{-}}$ semigroup $\{T(t): t \geq 0\}, F: J \times E \rightarrow P_{c k}(E)$ a multifunction, $g: P C(J, E) \rightarrow E$, and $J_{i}: E \rightarrow E(J=1,2, \ldots, m)$. We assume the following conditions.

(HA) The $C_{0}$-semigroup $\{T(t): t \geq 0\}$ is equicontinuous; that is, for any $x \in E, \lim _{t \rightarrow s}\|T(t) x-T(s) x\|=0$, independently of $x$.

(HF1) For every $x \in E, t \rightarrow F(t, x)$ is measurable, for every $t \in J, x \rightarrow F(t, x)$ is upper semicontinuous, and for every $z \in P C(J, E)$, the set $S_{F(\cdot z(\cdot))}^{1}:=\left\{f \in L^{1}(J, E)\right.$ : $f(t) \in F(t, z(t))$ a.e.\} is nonempty.

(HF2) There exists a function $\varphi \in L^{1 / q}\left(J, \mathbb{R}^{+}\right), q \in(0, \alpha)$ and a nondecreasing continuous function $\Omega: \mathbb{R}^{+} \rightarrow \mathbb{R}^{+}$ such that for any $x \in E$

$$
\|F(t, x)\| \leq \varphi(t) \Omega(\|x\|), \quad \text { a.e. } t \in J .
$$

(HF3) There exists a function $\beta \in L^{1 / q}\left(J, \mathbb{R}^{+}\right), q \in(0, \alpha)$ satisfying

$$
2 \rho\|\beta\|_{L^{1 / q\left(J, \mathbb{R}^{+}\right)}}<1,
$$

and for every bounded subset $D \subseteq E, \chi(F(t, D)) \leq$ $\beta(t) \chi(D)$, for a.e. $t \in J$, where $\chi$ is the Hausdorff measure of noncompactness in $E, \rho=M b^{\alpha-q} / \Gamma(\alpha)(\omega+$ $1)^{1-q}$ and $\omega=(\alpha-1) /(1-q)$.

(Hg) The function $g$ is continuous, compact, and there are two positive constants $a, d$ such that

$$
\|g(x)\| \leq a\|x\|+d, \quad \forall x \in P C(J, E) .
$$

(HI) For every $i=1,2, \ldots, m, I_{i}$ is continuous and compact and there exists a positive constant $h_{i}$ such that

$$
\left\|I_{i}(x)\right\| \leq h_{i}\|x\|, \quad x \in E .
$$


Then the problem (1) has a mild solution provided that there is $r>0$ such that

$$
M\left(\left\|x_{0}\right\|+a r+d+h r+\eta \Omega(r)\right) \leq r,
$$

where $h=\sum_{i=1}^{i=m} h_{i}, \eta=(\rho / M)\|\varphi\|_{L^{1 / q\left(J, \mathbb{R}^{+}\right)}}$and $M>0$ are such that $\sup _{t \geq 0}\|T(t)\| \leq M$.

Proof. In view of (HF1) for every $x \in P C(J, E)$, the set $S_{F(\cdot, z(\cdot))}^{1}$ is nonempty. So, we can introduce the multifunction $R$ : $P C(J, E) \rightarrow 2^{P C(J, E)}$ which is defined as let $x \in P C(J, E)$. A function $y \in R(x)$ if and only if for each $J=0,1,2, \ldots, m$,

$$
y(t)=\left\{\begin{aligned}
K_{1}(t)\left(x_{0}-g(x)\right) & \\
+\int_{0}^{t}(t-s)^{\alpha-1} K_{2}(t-s) & \\
\quad \times f(s) d s, & \\
K_{1}(t)\left(x_{0}-g(x)\right) & \\
+\sum_{k=1}^{k=J} K_{1}\left(t-t_{k}\right) I_{k}\left(x\left(t_{k}^{-}\right)\right) & \\
+\int_{0}^{t}(t-s)^{\alpha-1} K_{2}(t-s) & \\
\times f(s) d s, & t \in J_{i}, i \geq 1
\end{aligned}\right.
$$

where $f \in S_{F(\cdot, x(\cdot))}^{1}$. It is easy to see that any fixed point for $R$ is a mild solution for (1). So our goal is to prove, by using Theorem 19, that $R$ has a fixed point. The proof will be given in several steps.

Step 1 (the values of $R$ are closed). Let $x \in P C(J, E)$ and $\left(y_{n}\right)$, $n \geq 1$ be a sequence in $R(x)$ such that $y_{n} \rightarrow y$ in $P C(J, E)$. Then, according to the definition of $R$, there is a sequence $\left(f_{n}\right)_{n \geq 1}$, in $S_{F(\cdot, x(\cdot))}^{1}$ such that for any $t \in J_{i}, i=0,1, \ldots, m$,

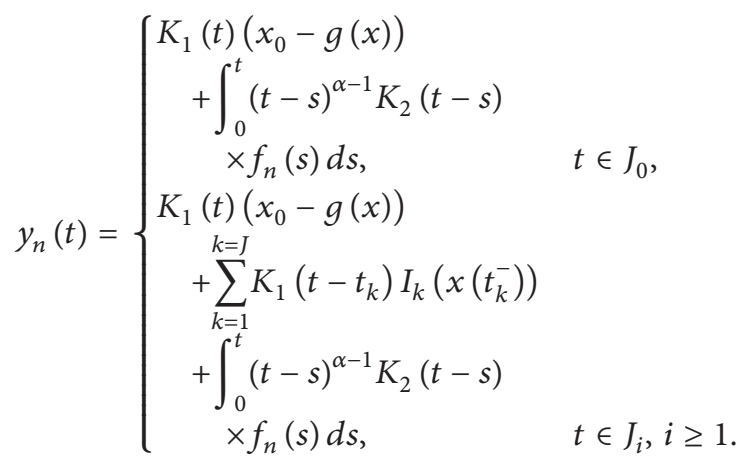

In view of (25) for every $n \geq 1$ and for a.e. $t \in J$,

$$
\left\|f_{n}(t)\right\| \leq \varphi(t) \Omega\left(\|x\|_{P C(J, E)}\right) .
$$

This shows that the set $\left\{f_{n}: n \geq 1\right\}$ is integrably bounded. Moreover, because $\left\{f_{n}(t): n \geq 1\right\} \subset F(t, x(t))$, for a.e. $t \in J$, the set $\left\{f_{n}(t): n \geq 1\right\}$ is relativity compact in $E$ for a.e. $t \in J$. Therefore, the set $\left\{f_{n}: n \geq 1\right\}$ is semicompact and then, by Lemma 6 it is weakly compact in $L^{1}\left(J, \mathbb{R}^{+}\right)$. So, without loss of generality we can assume that $f_{n}$ converges weakly to a function $f \in L^{1}\left(J, \mathbb{R}^{+}\right)$. From Mazur's lemma, for every natural number $j$ there is a natural number $k_{0}(j)>j$ and a sequence of nonnegative real numbers $\lambda_{j, k}$, $k=k_{0}(j), \ldots, j$ such that $\sum_{k=j}^{k_{0}} \lambda_{j, k}=1$, and the sequence of convex combinations $z_{j}=\sum_{k=j}^{k_{0}} \lambda_{j, k} f_{k}, j \geq 1$ converges strongly to $f$ in $L^{1}\left(J, \mathbb{R}^{+}\right)$as $j \rightarrow \infty$. Since $F$ takes convex values, using Lemma 6 , we obtain for a.e. $t \in J$,

$$
\begin{aligned}
f(t) & \in \bigcap_{j \geq 1} \overline{\left\{z_{k}(t): k \geq j\right\}} \\
& \subseteq \bigcap_{j \geq 1} \overline{\mathrm{CO}}\left\{f_{k}: k \geq j\right\} \subseteq F(t, x(t)) .
\end{aligned}
$$

Note that, by Lemma 15(iii), for every $t \in J, s \in(0, t]$ and every $n \geq 1$,

$$
\begin{aligned}
& |t-s|^{\alpha-1}\left\|K_{2}(t-s) f_{n}(s)\right\| \\
& \quad \leq|t-s|^{\alpha-1} \frac{M}{\Gamma(\alpha)} \varphi(t)\left(1+\|x\|_{P C(J, E)}\right) \in L^{1}\left((0, t], \mathbb{R}^{+}\right) .
\end{aligned}
$$

Therefore, by passing to the limit as $n \rightarrow \infty$ in (31), we obtain from the Lebesgue dominated convergence theorem that, for every $i=0,1, \ldots, m$,

$$
y(t)=\left\{\begin{array}{rr}
K_{1}(t)\left(x_{0}-g(x)\right) & \\
+\int_{0}^{t}(t-s)^{\alpha-1} K_{2}(t-s) & \\
\quad \times f(s) d s, & \\
K_{1}(t)\left(x_{0}-g(x)\right) & \\
+\sum_{k=1}^{k=J} K_{1}\left(t-t_{k}\right) J_{k}\left(x\left(t_{k}^{-}\right)\right) & \\
+\int_{0}^{t}(t-s)^{\alpha-1} K_{2}(t-s) & \\
\times f(s) d s, & t \in J_{i}, i \geq 1 .
\end{array}\right.
$$

This proves that $R(x)$ is closed.

Step 2. Let $B_{0}=\{x \in P C(J, E):\|x\| \leq r\}$. Obviously, $B_{0}$ is a bounded, closed and convex subset of $P C(J, E)$. We claim that $R\left(B_{0}\right) \subseteq B_{0}$.

To prove that, let $x \in B_{0}$ and $y \in R(x)$. For $t \in J_{0}$. By using Lemma 15, (25), (Hg), and Hölder's inequality we get for $t \in J_{0}$,

$$
\begin{aligned}
& \|y(t)\| \\
& \quad \leq M\left(\left\|x_{0}\right\|+a r+d\right)+\frac{M}{\Gamma(\alpha)} \Omega(r) \int_{0}^{t}(t-s)^{\alpha-1} \varphi(s) d s \\
& \quad \leq M\left(\left\|x_{0}\right\|+a r+d\right)+\frac{b^{\alpha-q} M \Omega(r)}{\Gamma(\alpha)(\omega+1)^{1-q}}\|\varphi\|_{L^{1 / q}\left(J, \mathbb{R}^{+}\right)} \\
& \quad \leq M\left(\left\|x_{0}\right\|+a r+d+\Omega(r) \eta\right) \leq r .
\end{aligned}
$$


Similarly, we obtain

$$
\|y(t)\| \leq M\left(\left\|x_{0}\right\|+a r+d+h r+\eta \Omega(r)\right) \leq r .
$$

Therefore, $R\left(B_{r}\right) \subseteq B_{r}$.

Step 3. Let $Z=R\left(B_{0}\right)$. We claim that the set $Z_{\mid \overline{J_{i}}}$ is equicontinuous for every $i=0,1,2, \ldots, m$, where

$$
\begin{gathered}
Z_{\mid \bar{J}_{i}}=\left\{y^{*} \in C\left(\overline{J_{i}}, E\right): y^{*}(t)=y(t), t \in J_{i}=\left(t_{i}, t_{i+1}\right],\right. \\
\left.y^{*}\left(t_{i}\right)=y\left(t_{i}^{+}\right), y \in Z\right\} .
\end{gathered}
$$

Let $y \in Z$. Then there is $x \in B_{r}$ with $y \in R(x)$. By recalling the definition of $R$, there is $f \in S_{F(\cdot, x(\cdot))}^{1}$ such that

$$
y(t)=\left\{\begin{array}{l}
K_{1}(t)\left(x_{0}-g(x)\right) \\
+\int_{0}^{t}(t-s)^{\alpha-1} K_{2}(t-s) \\
\times f(s) d s, \\
K_{1}(t)\left(x_{0}-g(x)\right) \\
k=J \\
+\sum_{k=1}^{k} K_{1}\left(t-t_{k}\right) J_{k}\left(x\left(t_{k}^{-}\right)\right) \\
+\int_{0}^{t}(t-s)^{\alpha-1} K_{2}(t-s) \\
\quad \times f(s) d s, \\
\end{array} \quad t \in J_{i}, i \geq 1 .\right.
$$

We consider the following cases.

Case 1 . When $i=0$, let $t, t+\lambda$ be two points in $\overline{J_{0}}=J_{0}$; then

$$
\left\|y^{*}(t+\lambda)-y^{*}(t)\right\| \leq G_{1}+G_{2}+G_{3}+G_{4},
$$

where

$$
\begin{gathered}
G_{1}=\left\|K_{1}(t+\lambda)\left(x_{0}-g(x)\right)-K_{1}(t)\left(x_{0}-g(x)\right)\right\|, \\
G_{2}=\left\|\int_{t}^{t+\lambda}(t+\lambda-s)^{\alpha-1} K_{2}(t+\lambda-s) f(s) d s\right\|, \\
G_{3}=\left\|\int_{0}^{t}\left[(t+\lambda-s)^{\alpha-1}-(t-s)^{\alpha-1}\right] K_{2}(t+\lambda-s) f(s) d s\right\|, \\
G_{4}=\left\|\int_{0}^{t}(t-s)^{\alpha-1}\left[K_{2}(t+\lambda-s)-K_{2}(t-s)\right] f(s) d s\right\| .
\end{gathered}
$$

We only need to check $G_{i} \rightarrow 0$ as $\lambda \rightarrow 0$ for every $i=$ $1,2,3,4$. By the equicontinuity of $\{T(t): t \in J\}$ we have

$$
\begin{gathered}
\lim _{\lambda \rightarrow 0} G_{1} \\
=\lim _{\lambda \rightarrow 0}\left\|K_{1}(t+\lambda)\left(x_{0}-g(x)\right)-K_{1}(t)\left(x_{0}-g(x)\right)\right\| \\
\leq \lim _{\lambda \rightarrow 0} \| \int_{0}^{\infty} \xi_{\alpha}(\theta) T\left((t+\lambda)^{\alpha} \theta\right)\left(x_{0}-g(x)\right) d \theta \\
\quad-\int_{0}^{\infty} \xi_{\alpha}(\theta) T\left(t^{\alpha} \theta\right)\left(x_{0}-g(x)\right) d \theta \| \\
\leq \lim _{\lambda \rightarrow 0} \int_{0}^{\infty} \xi_{\alpha}(\theta) \| T\left((t+\lambda)^{\alpha} \theta\right)\left(x_{0}-g(x)\right) \\
\quad-T\left(t^{\alpha} \theta\right)\left(x_{0}-g(x)\right) \| d \theta=0,
\end{gathered}
$$

independently of $x$.

For $G_{2}$ and $G_{3}$, one can repeat the same procedure in Theorem 4 of [22] to obtain

$$
\begin{aligned}
& \lim _{\lambda \rightarrow 0} G_{2}=0, \\
& \lim _{\lambda \rightarrow 0} G_{3}=0,
\end{aligned}
$$

independently of $x \in B_{r}$.

For $G_{4}$, by using (HA) and the Lebesgue dominated convergence theorem, we get

$$
\begin{aligned}
& \lim _{\lambda \rightarrow 0} G_{4} \\
& \leq \int_{0}^{t} \int_{0}^{\infty} \theta(t-s)^{\alpha-1} \zeta_{\alpha}(\theta) \\
& \quad \times\left[\lim _{\lambda \rightarrow 0} \| T\left((t+\lambda-s)^{\alpha} \theta\right) f(s)\right. \\
& \left.\quad-T\left((t-s)^{\alpha} \theta\right) f(s) \|\right] d \theta d s=0,
\end{aligned}
$$

independently of $f \in S_{F(\cdot, x(\cdot))}^{1}$ and $x \in B_{r}$.

Case 2. When $i \in\{1,2, \ldots, m\}$, let $t, t+\lambda$ be two points in $J_{i}$. Invoking to the definition of $R$, we have

$$
\begin{aligned}
& \left\|y^{*}(t+\lambda)-y^{*}(t)\right\| \\
& =\|y(t+\lambda)-y(t)\| \\
& \leq\left\|K_{1}(t+\lambda)\left(x_{0}-g(x)\right)-K_{1}(t)\left(x_{0}-g(x)\right)\right\| \\
& \quad+\sum_{k=1}^{k=i} \| K_{1}\left(t+\lambda-t_{k}\right) J_{k}\left(x\left(t_{k}^{-}\right)\right) \\
& \quad-K_{1}\left(t-t_{k}\right) J_{k}\left(x\left(t_{k}^{-}\right)\right) \| \\
& \quad \| \int_{0}^{t+\lambda}(t+\lambda-s)^{\alpha-1} k_{2}(t+\lambda-s) f(s) d s \\
& \quad-\int_{0}^{t}(t-s)^{\alpha-1} k_{2}(t-s) f(s) d s \| .
\end{aligned}
$$


Arguing as in the first case, we get

$$
\lim _{\lambda \rightarrow 0}\|y(t+\lambda)-y(t)\|=0 .
$$

Case 3. When $t=t_{i}, i=1, \ldots, m$, let $\lambda>0$ be such that $t_{i}+\lambda \in J_{i}$ and $\sigma>0$ such that $t_{i}<\sigma<t_{i}+\lambda \leq t_{i+1}$, then we have

$$
\left\|y^{*}\left(t_{i}+\lambda\right)-y^{*}\left(t_{i}\right)\right\|=\lim _{\sigma \rightarrow t_{i}^{+}}\left\|y\left(t_{i}+\lambda\right)-y(\sigma)\right\| .
$$

According the definition of $R$, we get

$$
\begin{aligned}
& \left\|y\left(t_{i}+\lambda\right)-y(\sigma)\right\| \\
& \leq\left\|k_{1}\left(t_{i}+\lambda\right)\left(x_{0}-g(x)\right)-k_{1}(\sigma)\left(x_{0}-g(x)\right)\right\| \\
& +\sum_{k=1}^{k=J} \| K_{1}\left(t_{i}+\lambda-t_{k}\right) J_{k}\left(x\left(t_{k}^{-}\right)\right) \\
& +\| \int_{0}^{t_{i}+\lambda}\left(t_{i}+\lambda-s\right)^{\alpha-1} k_{2}\left(t_{i}+\lambda-s\right) f(s) d s \\
& \quad-\int_{0}^{\sigma}(\sigma-s)^{\alpha-1} k_{2}(\sigma-s) f(s) d s \| .
\end{aligned}
$$

Arguing as in the first case, we can see that

$$
\lim _{\substack{\lambda \rightarrow 0, \sigma \rightarrow t_{i}^{+}}}\left\|y\left(t_{i}+\lambda\right)-y(\sigma)\right\|=0 .
$$

From (40)-(49) we conclude that $Z_{\mid \overline{J_{i}}}$ is equicontinuous for every $i=0,1,2, \ldots, m$.

Now for every $n \geq 1$, set $B_{n}=\overline{\operatorname{conv}} R\left(B_{n-1}\right)$. From Step $1, B_{n}$ is a nonempty, closed, and convex subset of $P C(J, E)$. Moreover, $B_{1}=\overline{\operatorname{conv}} R\left(B_{0}\right) \subseteq B_{0}$. Also $B_{2}=\overline{\operatorname{conv}} R\left(B_{1}\right) \subseteq$ $\overline{\operatorname{conv}} R\left(B_{0}\right) \subseteq B_{1}$. By induction, the sequence $\left(B_{n}\right), n \geq 1$ is a decreasing sequence of nonempty, closed, and bounded subsets of $P C(J, E)$. Our goal is to show that the subset $B=$ $\bigcap_{n=1}^{\infty} B_{n}$ is nonempty and compact in $P C(J, E)$.

By Lemma 7 (generalized Cantor's intersection), it is enough to show that

$$
\lim _{n \rightarrow \infty} \chi_{P C}\left(B_{n}\right)=0,
$$

where $\chi_{P C}$ is the Hausdorff measure of noncompactness on $P C(J, E)$ defined in Section 2.

In the following step we prove (50).

Step 4. Our aim in this step is to show that Relation (50) is satisfied. Let $n \geq 1$ be a fixed natural number and $\varepsilon>0$. In view of Lemma 9 , there exists a sequence $\left(y_{k}\right), k \geq 1$ in $R\left(B_{n-1}\right)$ such that

$$
\chi_{P C}\left(B_{n}\right)=\chi_{P C} R\left(B_{n-1}\right) \leq 2 \chi_{P C}\left\{y_{k}: k \geq 1\right\}+\varepsilon .
$$

From the definition of $\chi_{P C}$, the above inequality becomes

$$
\chi_{P C}\left(B_{n}\right) \leq 2 \max _{i=0,1, \ldots, m} \chi_{i}\left(S_{\mid \overline{J_{i}}}\right)+\varepsilon
$$

where $S=\left\{y_{k}: k \geq 1\right\}$ and $\chi_{i}$ is the Hausdorff measure of noncompactness on $C\left(\overline{J_{i}}, E\right)$. Arguing as in the previous step we can show that $B_{n \mid \bar{J}_{i}}, J=0,1, \ldots, m$, is equicontinuity. Then, by applying Lemma 8 , we obtain

$$
\chi_{i}\left(S_{\mid \overline{J_{i}}}\right)=\sup _{t \in \overline{J_{i}}} \chi(S(t))
$$

where $\chi$ is the Hausdorff measure of noncompactness on $E$. Therefore, by using the nonsingularity of $\chi$, (52) becomes

$$
\begin{aligned}
\chi_{P C}\left(B_{n}\right) & \leq 2 \max _{i=0,1, \ldots, m}\left\{\sup _{t \in \overline{J_{i}}} \chi(S(t))\right\}+\epsilon \\
& =2 \sup _{t \in J} \chi(S(t))+\varepsilon \\
& =2 \sup _{t \in J} \chi\left\{y_{k}(t): k \geq 1\right\}+\varepsilon .
\end{aligned}
$$

Now, since $y_{k} \in R\left(B_{n-1}\right), k \geq 1$, there is $x_{k} \in B_{n-1}$ such that $y_{k} \in R\left(x_{k}\right), k \geq 1$. By recalling the definition of $R$ for every $k \geq 1$ there is $f_{k} \in S_{F\left(\cdot, x_{k}(\cdot)\right)}^{1}$ such that for every $t \in J$,

$$
\begin{aligned}
& \chi\left\{y_{k}(t): k \geq 1\right\}
\end{aligned}
$$

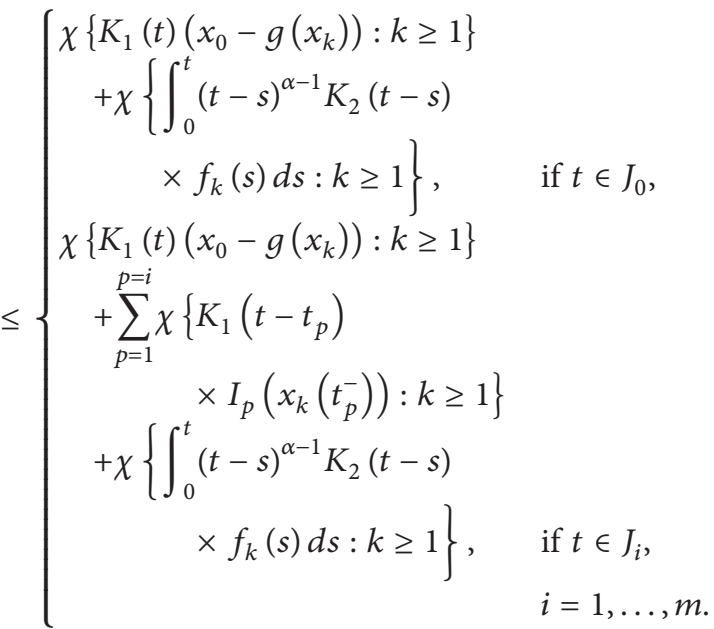

Since $g$ is compact, the set $\left\{g\left(x_{k}\right): k \geq 1\right\}$ is relatively compact. Hence, for every $t \in J$, we have

$$
\chi\left\{k_{1}(t)\left(x_{0}-g\left(x_{k}\right)\right): k \geq 1\right\}=0 .
$$

Furthermore, condition (HI) implies, for every $p=$ $1,2, \ldots, m$ and every $t \in J$,

$$
\chi\left\{k_{1}\left(t-t_{p}\right)\left(I_{p}\left(x_{k}\left(t_{p}^{-}\right)\right)\right): k \geq 1\right\}=0 .
$$

In order to estimate the quantity $\chi\left\{\int_{0}^{t}(t-s)^{\alpha-1} K_{2}(t-\right.$ s) $\left.f_{k}(s) d s: k \geq 1\right\}$, we consider the linear continuous map

$$
\Pi: L^{1 / q}(J, E) \longrightarrow C(J, E),
$$

where

$$
\Pi(f)(t)=\int_{0}^{t}(t-s)^{\alpha-1} k_{2}(t-s) f(s) d s
$$


Using Hölder's inequality to obtain for any $f_{1}, f_{2} \in$ $L^{1 / q}(J, E)$ and any $t \in J$,

$$
\begin{aligned}
& \left\|\Pi\left(f_{1}\right)(t)-\Pi\left(f_{2}\right)(t)\right\| \\
& \quad \leq \frac{M}{\Gamma(\alpha)} \int_{0}^{t}(t-s)^{\alpha-1}\left\|f_{1}(s)-f_{2}(s)\right\| d s \\
& \quad \leq \frac{M}{\Gamma(\alpha)}\left\|f_{1}-f_{2}\right\|_{L^{1 / q}\left(J, \mathbb{R}^{+}\right)}\left(\int_{0}^{t}(t-s)^{(\alpha-1) /(1-q)} d s\right)^{1-q} \\
& \quad \leq \rho\left\|f_{1}-f_{2}\right\|_{L^{1 / q}\left(J, \mathbb{R}^{+}\right)} .
\end{aligned}
$$

Furthermore, by $\left(\mathrm{H}_{4}\right)$, for almost $t \in J,\left\|f_{k}(t)\right\| \leq \varphi(t) \Omega(r)$. Consequently, $f_{k} \in L^{1 / q}(J, E), k \geq 1$.

We observe that, from $\left(\mathrm{H}_{5}\right)$ it holds that for a.e. $t \in J$,

$$
\begin{aligned}
\chi\left\{f_{k}(t): k \geq 1\right\} & \leq \chi\left\{F\left(s, x_{k}(t)\right): k \geq 1\right\} \\
& \leq \beta(t) \chi\left\{x_{k}(t): k \geq 1\right\} \\
& \leq \beta(t) \chi\left(B_{n-1}(t)\right) \\
& \leq \beta(t) \chi_{P C}\left(B_{n-1}\right)=\gamma(t) .
\end{aligned}
$$

Note that $\gamma \in L^{1 / q}\left(J, \mathbb{R}^{+}\right)$. Then, by virtue of Lemma 10, there exists a compact $K_{\epsilon} \subseteq E$, a measurable set $J_{\epsilon} \subset J$, with measure less than $\epsilon$ and a sequence of functions $\left\{g_{k}^{\epsilon}\right\} \subset$ $L^{1 / q}(J, E)$ such that for all $s \in J,\left\{g_{k}^{\epsilon}(s): k \geq 1\right\} \subseteq K_{\epsilon}$, and

$$
\begin{array}{ll}
\left\|f_{k}(s)-g_{k}^{\epsilon}(s)\right\|<2 \gamma(s)+\epsilon, & \text { for every } k \geq 1 \\
& \text { and every } s \in J-J_{\epsilon} .
\end{array}
$$

Using (60) and (62), to obtain for all $t \in J$ and all $k \geq 1$

$$
\begin{aligned}
& \left\|\Pi\left(z_{k}\right)(t)-\Pi\left(g_{k}^{\epsilon}\right)(t)\right\| \\
& \leq \rho\left\|f_{k}-g_{k}^{\epsilon}\right\|_{L^{1 / q}\left(J, \mathbb{R}^{+}\right)} \\
& =\rho\left[\int_{J-J_{\epsilon}}\left\|f_{k}(s)-g_{k}^{\epsilon}(s)\right\|^{1 / q} d s\right. \\
& \left.\quad+\int_{J_{\epsilon}}\left\|f_{k}(s)-g_{k}^{\epsilon}(s)\right\|^{1 / q} d s\right]^{q} \\
& \leq \rho\left[\int_{J-J_{\epsilon}}(2 \gamma(s)+\epsilon)^{1 / q} d s+\int_{J_{\epsilon}}\left\|f_{k}(s)-g_{k}^{\epsilon}(s)\right\|^{1 / q} d s\right]^{q} .
\end{aligned}
$$

By taking into account that $\varepsilon$ is arbitrary, we get for all $t \in J$ and all $k \geq 1$

$$
\begin{aligned}
\left\|\Pi\left(f_{k}\right)(t)-\Pi\left(g_{k}^{\epsilon}\right)(t)\right\| & \leq 2 \rho \int_{0}^{b} \gamma(s) d s \\
& =2 \rho \chi_{P C}\left(B_{n-1}\right)\|\beta\|_{L^{1 / q}\left(J, \mathbb{R}^{+}\right.} .
\end{aligned}
$$

Therefore, for all $t \in J$,

$$
\begin{aligned}
\chi\left(\left\{\int_{0}^{t}(t-s)^{\alpha-1} k_{2}(t-s) f_{k}(s) d s: k \geq 1\right\}\right) \\
=\chi\left\{\Pi\left(f_{k}\right)(t): k \geq 1\right\} \\
\quad \leq \chi\left\{\Pi\left(g_{k}^{\epsilon}\right)(t): k \geq 1\right\} \\
\quad+\chi\left\{\Pi\left(f_{k}\right)(t)-\Pi\left(g_{k}^{\epsilon}\right)(t): k \geq 1\right\} \\
\quad \leq \chi\left\{\Pi\left(f_{k}\right)(t)-\Pi\left(g_{k}^{\epsilon}\right)(t): k \geq 1\right\} \\
\quad \leq 2 \rho \chi_{P C}\left(B_{n-1}\right)\|\beta\|_{L^{1 / q}\left(J, \mathbb{R}^{+}\right)} .
\end{aligned}
$$

Then, by (55)-(57) and (65) for every $t \in J$,

$$
\chi\left\{y_{k}(t): k \geq 1\right\} \leq 2 \rho \chi_{P C}\left(B_{n-1}\right)\|\beta\|_{L^{1 / q\left(J, \mathbb{R}^{+}\right)}} .
$$

This inequality with (54) and with the fact that $\varepsilon$ is arbitrary, implies

$$
\chi_{P C}\left(B_{n}\right) \leq 2 \rho \chi_{P C}\left(B_{n-1}\right)\|\beta\|_{\left.L^{1 / q\left(J, \mathbb{R}^{+}\right.}\right)} .
$$

By means of a finite number of steps, we can write

$$
0 \leq \chi_{P C}\left(B_{n}\right) \leq\left(2 \rho\|\beta\|_{L^{1 / q}\left(J, \mathbb{R}^{+}\right)}\right)^{n-1} \chi_{P C}\left(B_{1}\right), \quad \forall n \geq 1 .
$$

Since this inequality is true for every $n \in \mathbb{N}$, by (26) and by passing to the limit as $n \rightarrow+\infty$, we obtain (50), and so our aim in this step is verified.

At this point, we are in position to apply the generalized Cantor's intersection property (Lemma 7) and claim that the set $B=\bigcap_{n=1}^{\infty} B_{n}$ is a nonempty and compact subset of $P C(J, E)$. Moreover, for every $B_{n}$ being bounded, closed, and convex, $B$ is also bounded, closed, and convex.

Step 5 . Let us verify that $R(B) \subseteq B$.

Indeed, $R(B) \subseteq R\left(B_{n}\right) \subseteq \overline{\operatorname{conv}} R\left(B_{n}\right)=B_{n+1}$, for every $n \geq 1$. Therefore, $R(B) \subset \bigcap_{n=2}^{\infty} B_{n}$. On the other hand $B_{n} \subset B_{1}$ for every $n \geq 1$. So, $R(B) \subset \bigcap_{n=2}^{\infty} B_{n}=\bigcap_{n=1}^{\infty} B_{n}=B$.

Step 6. The graph of the multivalued function $R_{\mid B}: B \rightarrow 2^{B}$ is closed.

Consider a sequence $\left\{x_{n}\right\}_{n \geq 1}$ in $B$ with $x_{n} \rightarrow x$ in $B$ and let $y_{n} \in R\left(x_{n}\right)$ with $y_{n} \rightarrow y$ in $P C(J, E)$. We have to show that $y \in R(x)$. By recalling the definition of $R$, for any $n \geq 1$, there is $f_{n} \in S_{F\left(\cdot, x_{n}(\cdot)\right)}^{1}$ such that

$$
y_{n}(t)=\left\{\begin{array}{c}
K_{1}(t)\left(x_{0}-g\left(x_{n}\right)\right) \\
+\int_{0}^{t}(t-s)^{\alpha-1} K_{2}(t-s) \\
\times f_{n}(s) d s, \\
K_{1}(t)\left(x_{0}-g\left(x_{n}\right)\right) \\
+\sum_{k=1}^{k=i} K_{1}\left(t-t_{k}\right) I_{k}\left(x\left(t_{k}^{-}\right)\right) \\
+\int_{0}^{t}(t-s)^{\alpha-1} K_{2}(t-s) \\
\quad \times f_{n}(s) d s,
\end{array} \quad t \in J_{i}, i \geq 1 .\right.
$$


Observe that for every $n \geq 1$ and for a.e. $t \in J$,

$$
\begin{aligned}
\left\|f_{n}(t)\right\| & \leq \varphi(t)\left(1+\Omega\left(\left\|y_{n}\right\|_{P C(J, E)}\right)\right) \\
& \leq \varphi(t)(1+\Omega(r)) .
\end{aligned}
$$

This shows that the set $\left\{f_{n}: n \geq 1\right\}$ is integrably bounded. In addition, the set $\left\{f_{n}(t): n \geq 1\right\}$ is relatively compact for a.e. $t \in J$ because assumption (HF3) both with the convergence of $\left\{y_{n}\right\}_{n \geq 1}$ implies that

$$
\begin{aligned}
\chi\left\{f_{n}(t): n \geq 1\right\} & \leq \chi F\left(t,\left\{x_{n}(t): n \geq 1\right\}\right) \\
& \leq \beta(t) \chi\left\{x_{n}(t): n \geq 1\right\}=0 .
\end{aligned}
$$

Hence, the sequence $\left\{f_{n}\right\}_{n \geq 1}$ is semicompact; hence, by Lemma 4 , it is weakly compact in $L^{1}(J, E)$. So, without loss of generality we can assume that $f_{n}$ converges weakly to a function $f \in L^{1}\left(J, \mathbb{R}^{+}\right)$. From Mazur's lemma, for every natural number $j$, there are a natural number $k_{0}(j)>j$ and a sequence of nonnegative real numbers $\lambda_{j, k}, k=$ $k_{0}(j), \ldots, j$ such that $\sum_{k=j}^{k_{0}} \lambda_{j, k}=1$ and the sequence of convex combinations $z_{j}=\sum_{k=j}^{k_{0}} \lambda_{j, k} f_{k}, j \geq 1$, converges strongly to $f$ in $L^{1}\left(J, \mathbb{R}^{+}\right)$as $j \rightarrow \infty$. Since $F$ takes convex values, using Lemma 6 , we get for a.e. $t \in J$,

$$
\begin{aligned}
f(t) & \in \bigcap_{j \geq 1} \overline{\left\{z_{k}(t): k \geq j\right\}} \subseteq \bigcap_{j \geq 1} \overline{\operatorname{conv}}\left\{\bigcup_{k \geq j} F\left(t, x_{k}(t)\right)\right\} \\
& =\overline{\text { conv }} \lim _{k \rightarrow \infty} \sup F\left(t, x_{k}(t)\right) .
\end{aligned}
$$

Since $F$ is upper semicontinuous with compact values, $\lim _{k \rightarrow \infty} \sup F\left(t, x_{k}(t)\right) \subseteq F(t, x(t))$. Because the values of $F$ are convex, we conclude that $f(t) \in F(t, x(t))$, a.e. $t \in J$. Then, by the continuity of $g, K_{1}, K_{2}, I_{k}(k=1,2, \ldots, m)$ and by the same arguments used in Step 1, we conclude from relation (69) that

$$
y(t)=\left\{\begin{aligned}
K_{1}(t)\left(x_{0}-g(x)\right) & \\
+\int_{0}^{t}(t-s)^{\alpha-1} K_{2}(t-s) & \\
\quad \times f(s) d s, & \\
K_{1}(t)\left(x_{0}-g(x)\right) & \\
+\sum_{k=1}^{k=J} K_{1}\left(t-t_{k}\right) I_{k}\left(x\left(t_{k}^{-}\right)\right) & \\
+\int_{0}^{t}(t-s)^{\alpha-1} K_{2}(t-s) & \\
\times f(s) d s, & t \in J_{i}, i \geq 1
\end{aligned}\right.
$$
closed.

Therefore, $y \in R(x)$. This shows that the graph of $R$ is

As a result of Steps $1-5$, the multivalued $R_{\mid B}: B \rightarrow 2^{B}$ is closed and $\chi$-condensing, with nonempty convex compact values. Applying Theorem 18, there is $x \in B$ such that $x \in$ $R(x)$. Clearly $x$ is a mild solution of (1).

To end this section, we prove that the set of mild solutions of (1) is compact.
Theorem 21. If the function $\Omega$ in (HF2) is given of the form $\Omega(r)=r+1$, then under the assumptions (HA), (HF1), (HF3), $(\mathrm{Hg})$, and (HI) of Theorem 20, the set of mild solutions of (1) is compact in $P C(J, E)$ provided that

$$
M(a+h+\eta)<1
$$

Proof. Note that by Theorem 20, the set of solutions of (1) is nonempty. Assume that $x$ is a mild solution of (1). Then

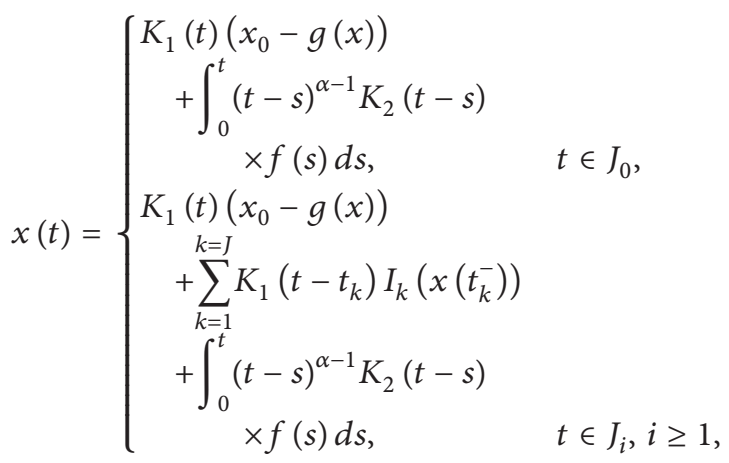

where $f$ is an integrable selection for $F(\cdot, x(\cdot))$.

By arguing as in Step 2 of Theorem 20 we get

$$
\begin{aligned}
& \|x\|_{P C(J, E)} \\
& \leq M\left(\left\|x_{0}\right\|+a\|x\|_{P C(J, E)}+d\right. \\
& \left.+h\|x\|_{P C(J, E)}+\eta\left(\|x\|_{P C(J, E)}+1\right)\right) \text {. }
\end{aligned}
$$

Therefore,

$$
\|x\|_{P C(J, E)} \leq \frac{M\left[\left\|x_{0}\right\|+d+\eta\right]}{1-M(a+h+\eta)}
$$

This inequality with (74) yields that the set of solutions is bounded subset of $P C(J, E)$. Hence, by Theorem 18, it is compact.

\section{Application to Controllability}

In this section we use the methods in the above section to discuss the controllability of (4) and (5).

Now, we suppose that $E$ is a real separable Banach space and $X$ is a real Banach space. 
Definition 22. A function $x \in P C(J, E)$ is said to be a mild solution for (4) if there exists an integrable selection $f$ for $F(\cdot, x(\cdot))$ such that for each $t \in J$,

$$
x(t)=\left\{\begin{aligned}
K_{1}(t)\left(x_{0}-g(x)\right) & \\
& +\int_{0}^{t}(t-s)^{\alpha-1} K_{2}(t-s) \\
& \times(f(s)+B(u(s))) d s, \quad t \in J_{0}, \\
K_{1}(t)\left(x_{0}-g(x)\right) & \\
+\sum_{k=1}^{k=i} K_{1}\left(t-t_{k}\right) I_{k}\left(x\left(t_{k}^{-}\right)\right) & \\
+\int_{0}^{t}(t-s)^{\alpha-1} K_{2}(t-s) & \times(f(s)+B(u(s))) d s, \quad t \in J_{i}, i \geq 1 .
\end{aligned}\right.
$$

Definition 23. The system (4) is said to be nonlocally controllable on the interval $J$ if for every $x_{0}, x_{1} \in E$, there exists a control function $u \in L^{2}(J, X)$ such that a mild solution of (4) satisfies $x(0)=x_{0}-g(x), x(b)=x_{1}-g(x)$.

Theorem 24. Let $\alpha \in(1 / 2,1), B: X \rightarrow E$ be a bounded linear operator, $A$ the infinitesimal generator of a strongly semigroup $\{T(t): t \geq 0\}, F: J \times E \rightarrow P_{c k}(E)$ a multifunction, $g: P C(J, E) \rightarrow E$, and $I_{i}: E \rightarrow E(i=1,2, \ldots, m)$. We assume the following conditions.

(HF4) For every $x \in E, t \rightarrow F(t, x)$ is measurable.

(HF5) There is a function $\varsigma \in L^{1 / q}\left(J, \mathbb{R}^{+}\right), q \in(0, \alpha)$ such that

(i) for every $x, y \in E$

$h(F(t, x), F(t, y)) \leq \varsigma(t)\|x-y\|, \quad$ for a.e. $t \in J$

(ii) for every $x \in E$

$\sup \{\|x\|: x \in F(t, 0)\} \leq \varsigma(t), \quad$ for a.e. $t \in J$.

$(\mathrm{Hg})^{*}$ There is a positive constant a such that

$$
\|g(z)-g(w)\| \leq a\|z-w\|, \quad \forall z, w \in P C(J, E) .
$$

$(H I)^{*}$ For any $i=1,2, \ldots, m$, there is $h_{i}>0$ such that

$$
\left\|J_{i}(x)-J_{i}(y)\right\| \leq h_{i}\|x-y\|, \quad \forall x, y \in E .
$$

$(H W)$ The linear bounded operator $W: L^{2}(J, X) \rightarrow E$ defined by

$$
W(u)=\int_{0}^{b}(b-s)^{\alpha-1} K_{2}(b-s) B(u(s)) d s
$$

has an invertible $W^{-1}: E \rightarrow L^{2}(J, X) / \operatorname{Ker}(W)$, and there exists a positive constant $N$ such that $\left\|W_{i}^{-1}\right\| \leq N$ and $\|B\| \leq N$.
Then (4) is controllable on J provided

$$
\begin{aligned}
\gamma_{1}= & {\left[M(a+h+\delta)+\frac{N^{2}}{\Gamma(\alpha)}(a+M(a+\delta))\right] } \\
& \times \frac{b^{\alpha-1 / 2}}{\sqrt{2 \alpha-1}}<1,
\end{aligned}
$$

where $\delta=\left(b^{\alpha-q} / \Gamma(\alpha)(\omega+1)^{1-q}\right)\|\varsigma\|_{L^{1 / q}\left(J, \mathbb{R}^{+}\right)}$.

Proof. By (HF4) and (HF5)(i), we conclude from Lemma 2 that for any $z \in P C(J, E)$, there is an integrable selection for $F(\cdot, z(\cdot))$. Then, we can consider a multivalued $\widetilde{R}$ : $P C(J, E) \rightarrow 2^{P C(J, E)}$ as: let $z \in P C(J, E)$, a function $y \in \widetilde{R}(z)$ if and only if for each $t \in J$,

$$
y(t)=\left\{\begin{array}{cc}
K_{1}(t)\left(x_{0}-g(z)\right) & \\
& +\int_{0}^{t}(t-s)^{\alpha-1} K_{2}(t-s) \\
& \times\left(f(s)+B\left(u_{f}(s)\right)\right) d s, \quad t \in J_{0}, \\
K_{1}(t)\left(x_{0}-g(z)\right) & \\
& +K_{1}\left(t-t_{1}\right) I_{1}\left(z\left(t_{k}^{-}\right)\right) \\
& +\int_{0}^{t}(t-s)^{\alpha-1} K_{2}(t-s) \\
& \times\left(f(s)+B\left(u_{f}(s)\right)\right) d s, \quad t \in J_{1}, \\
\vdots & \\
K_{1}(t)\left(x_{0}-g(z)\right) & \\
& +\sum_{k=1}^{k=m} K_{1}\left(t-t_{k}\right) I_{k}\left(z\left(t_{k}^{-}\right)\right) \\
& +\int_{0}^{t}(t-s)^{\alpha-1} K_{2}(t-s)
\end{array}\right.
$$

where $f$ is an integrable selection for $F(\cdot, z(\cdot))$. Also, for any $z \in P C(J, E)$ and any integrable selection $f$ for $F(\cdot, z(\cdot))$, we define the control function $u_{f} \in L^{2}(J, X)$ by

$$
\begin{aligned}
u_{f}(t)=W^{-1}[ & x_{1}-g(z)-K_{1}(b)\left(x_{0}-g(z)\right) \\
& \quad-\sum_{i=1}^{i=m} K_{1}\left(b-t_{i}\right) I_{k}\left(z\left(t_{k}^{-}\right)\right) \\
& \left.-\int_{0}^{b}(b-s)^{\alpha-1} K_{2}(b-s) f(s) d s\right](t) .
\end{aligned}
$$

We will show that, when using this control, the multivalued function $\widetilde{R}$ has a fixed point. This fixed point is the desired 
mild solution. In fact, if $x$ is a fixed point for $\widetilde{R}$, then from (85) and (86) we get

$$
\begin{aligned}
x(b)= & K_{1}(b)\left(x_{0}-g(x)\right)+\sum_{k=1}^{k=m} K_{1}\left(b-t_{k}\right) I_{k}\left(x\left(t_{k}^{-}\right)\right) \\
& +\int_{0}^{b}(b-s)^{\alpha-1} K_{2}(b-s) f(s) d s \\
& +\int_{0}^{b}(b-s)^{\alpha-1} K_{2}(b-s) B\left(u_{f}(s)\right) d s \\
= & K_{1}(b)\left(x_{0}-g(x)\right)+\sum_{k=1}^{k=m} K_{1}\left(b-t_{k}\right) I_{k}\left(x\left(t_{k}^{-}\right)\right) \\
& +\int_{0}^{b}(b-s)^{\alpha-1} K_{2}(b-s) f(s) d s+W\left(u_{f}\right) \\
= & K_{1}(b)\left(x_{0}-g(x)\right)+\sum_{k=1}^{k=m} K_{1}\left(b-t_{k}\right) I_{k}\left(x\left(t_{k}^{-}\right)\right) \\
& +\int_{0}^{b}(b-s)^{\alpha-1} K_{2}(b-s) f(s) d s \\
& +\int_{0}^{b}(b-s)^{\alpha-1} K_{2}(b-s) f(s) d s \\
& +x_{1}-g(x)-K_{1}(b)\left(x_{0}-g(x)\right) \\
& -\sum_{k=m}^{k=m} K_{1}\left(b-t_{k}\right) I_{k}\left(x\left(t_{k}^{-}\right)\right) \\
& x_{1}^{b}(x) .
\end{aligned}
$$

Step 2 ( $\widetilde{R}$ is contraction). Take $z_{1}, z_{2} \in P C(J, E)$, and let $y_{1} \in$ $\widetilde{R}\left(z_{1}\right)$. Then, there is $f \in S_{F\left(\cdot, z_{1}(\cdot)\right)}^{1}$ such that for any $t \in J$,

$$
y_{1}(t)= \begin{cases}K_{1}(t)\left(x_{0}-g\left(z_{1}\right)\right) & \\ & +\int_{0}^{t}(t-s)^{\alpha-1} K_{2}(t-s) \\ & \times\left(f(s)+B\left(u_{f}(s)\right)\right) d s, \quad t \in J_{0}, \\ K_{1}(t)\left(x_{0}-g\left(z_{1}\right)\right) & \\ & +K_{1}\left(t-t_{1}\right) I_{1}\left(z_{1}\left(t_{1}^{-}\right)\right) \\ & +\int_{0}^{t}(t-s)^{\alpha-1} K_{2}(t-s) \\ & \times\left(f(s)+B\left(u_{f}(s)\right)\right) d s, \quad t \in J_{1}, \\ \vdots & \\ K_{1}(t)\left(x_{0}-g\left(z_{1}\right)\right) & \\ & +\sum_{k=1}^{k=m} K_{1}\left(t-t_{k}\right) I_{k}\left(z_{1}\left(t_{k}^{-}\right)\right) \\ & +\int_{0}^{t}(t-s)^{\alpha-1} K_{2}(t-s)\end{cases}
$$

Consider the multifunction $Z: J \rightarrow 2^{E}, Z(t)=\{u$ : $\left.\|f(t)-u\| \leq \varsigma(t)\left\|z_{1}(t)-z_{2}(t)\right\|\right\}$. In view of (HF5)(i), for any $t \in J, Z(t) \cap F\left(t, z_{2}(t)\right)$ is nonempty. Moreover, since the functions $\varsigma, z_{2}, z_{1}, f$ are measurable, the multifunction $t \rightarrow Z(t) \cap F\left(t, z_{2}(t)\right)$ is measurable with closed values (see [38]). Thus, there is $h \in S_{F\left(\cdot, z_{2}(\cdot)\right)}^{1}$ with

$$
\|h(t)-f(t)\| \leq \varsigma(t)\left\|z_{1}(t)-z_{2}(t)\right\|, \quad \text { a.e. } t \in J .
$$

Define assumptions of Theorem 19.

Step 1. The values of $\widetilde{R}$ are closed. In view of (HF5)(ii), for any $t \in J, x \in E$ and $y \in F(t, x)$, we have

$$
\begin{aligned}
\|y\| & \leq d(0, F(t, 0))+d(y, F(t, 0)) \\
& \leq \varsigma(t)+H(F(t, x(t)), F(t, 0)) \\
& \leq \varsigma(t)+\varsigma(t)\|x\|_{P C(J, E)} \\
& =\varsigma(t)\left(1+\|x\|_{P C(J, E)}\right) .
\end{aligned}
$$

Since $B$ is a linear bounded operator, we can follow the same arguments used in Step 1 of Theorem 20 to prove that the values of $\widetilde{R}$ are closed subset in $P C(J, E)$.

$$
y_{2}(t)=\left\{\begin{aligned}
K_{1}(t)\left(x_{0}-g\left(z_{2}\right)\right) & \\
& +\int_{0}^{t}(t-s)^{\alpha-1} K_{2}(t-s) \\
& \times\left(h(s)+B\left(u_{h}(s)\right)\right) d s, \quad t \in J_{0}, \\
K_{1}(t)\left(x_{0}-g\left(z_{2}\right)\right) & \\
& +K_{1}\left(t-t_{1}\right) I_{1}\left(z_{2}\left(t_{1}^{-}\right)\right) \\
& +\int_{0}^{t}(t-s)^{\alpha-1} K_{2}(t-s) \\
& \times\left(h(s)+B\left(u_{h}(s)\right)\right) d s, \quad t \in J_{1}, \\
\vdots & \\
K_{1}(t)\left(x_{0}-g\left(z_{2}\right)\right) & \\
& +\sum_{k=1}^{k=m} K_{1}\left(t-t_{k}\right) I_{k}\left(z_{2}\left(t_{k}^{-}\right)\right) \\
& +\int_{0}^{t}(t-s)^{\alpha-1} K_{2}(t-s)
\end{aligned}\right.
$$


Obviously $y_{2} \in \widetilde{R}\left(z_{2}\right)$. To simplify the calculation, we set

$$
\begin{aligned}
z^{f}= & x_{1}-g\left(z_{1}\right)-K_{1}(b)\left(x_{0}-g\left(z_{1}\right)\right) \\
& -\int_{0}^{b}(b-s)^{\alpha-1} K_{2}(b-s) f(s) d s, \\
z^{h}= & x_{1}-g\left(z_{2}\right)-K_{1}(b)\left(x_{0}-g\left(z_{2}\right)\right) \\
& -\int_{0}^{b}(b-s)^{\alpha-1} K_{2}(b-s) h(s) d s .
\end{aligned}
$$

Then, by (90) and $(\mathrm{Hg})^{*}$ we obtain

$$
\begin{aligned}
\| u_{f}- & u_{h} \|_{L^{2}(J, X)} \\
\leq & \left\|W^{-1}\left(z^{f}\right)-W^{-1}\left(z^{h}\right)\right\|_{L^{2}(J, X)} \\
\leq & \left\|W^{-1}\right\|\left\|z^{f}-z^{h}\right\|_{X} \\
\leq & N a\left\|z_{1}-z_{2}\right\|_{P C(J, E)}+M a\left\|z_{1}-z_{2}\right\|_{P C(J, E)} \\
& +\frac{M\left\|z_{1}-z_{2}\right\|_{P C(J, E)}}{\Gamma(\alpha)} \int_{0}^{b}(b-s)^{\alpha-1} \varsigma(s) d s \\
\leq & N\left[a\left\|z_{1}-z_{2}\right\|_{P C(J, E)}+M a\left\|z_{1}-z_{2}\right\|_{P C(J, E)}\right. \\
& \left.\quad+M \delta\left\|z_{1}-z_{2}\right\|_{P C(J, E)}\right] \\
\leq & \left\|z_{1}-z_{2}\right\|_{P C(J, E)} N[a+M a+M \delta] .
\end{aligned}
$$

This inequality with (89)-(90) yields for $t \in J_{0}$

$$
\begin{aligned}
\| y_{1}(t)- & y_{2}(t) \| \\
\leq & M\left\|g\left(z_{1}\right)-g\left(z_{2}\right)\right\|+\frac{M}{\Gamma(\alpha)} \int_{0}^{t}(t-s)^{\alpha-1} \\
& \times\|h(s)-f(s)\| d s+\frac{M}{\Gamma(\alpha)} \int_{0}^{t}(t-s)^{\alpha-1} \\
& \times\left\|B\left(u_{h}(s)\right)-B\left(u_{f}(s)\right)\right\| d s \\
\leq & M a\left\|z_{1}-z_{2}\right\|_{P C(J, E)}+\frac{M}{\Gamma(\alpha)} \int_{0}^{t}(t-s)^{\alpha-1} \varsigma(s) \\
& \times\left\|z_{1}-z_{2}\right\|_{P C(J, E)}+\frac{M N}{\Gamma(\alpha)} \int_{0}^{t}(t-s)^{\alpha-1} \\
& \times\left\|u_{h}(s)-u_{f}(s)\right\| d s \\
\leq & M a\left\|z_{1}-z_{2}\right\|_{P C(J, E)}+M \delta\left\|z_{1}-z_{2}\right\|_{P C(J, E)} \\
& +\frac{M N}{\Gamma(\alpha)}\left(\int_{0}^{t}(t-s)^{2(\alpha-1)} d s\right)^{1 / 2}\left\|u_{f}-u_{h}\right\|_{L^{2}\left(J_{0}, X\right)}
\end{aligned}
$$

$$
\begin{aligned}
\leq & \left\|z_{1}-z_{2}\right\|_{P C(J, E)} M(a+\delta) \\
& +\frac{M N}{\Gamma(\alpha)}\left\|z_{1}-z_{2}\right\|_{P C(J, E)} N[a+M a+M \delta] \frac{b^{\alpha-1 / 2}}{\sqrt{2 \alpha-1}} \\
\leq & \left\|z_{1}-z_{2}\right\|_{P C(J, E)} M\left[a+\delta+\frac{N^{2}}{\Gamma(\alpha)}(a+M a+M \delta)\right] \\
& \times \frac{b^{\alpha-1 / 2}}{\sqrt{2 \alpha-1}} \\
\leq & \left\|z_{1}-z_{2}\right\|_{P C(J, E)} .
\end{aligned}
$$

Similarly, for any $i=1,2, \ldots, m$, we get from (93) and conditions $(\mathrm{Hg})^{*}$ and $(\mathrm{HI})$,

$$
\begin{aligned}
\| y_{2}(t)- & y_{1}(t) \| \\
\leq & M\left\|z_{1}-z_{2}\right\|_{P C(J, E)}(a+h)+\frac{M}{\Gamma(\alpha)} \int_{0}^{t}(t-s)^{\alpha-1} \\
& \times\|h(s)-f(s)\| d s+\frac{M}{\Gamma(\alpha)} \int_{0}^{t}(t-s)^{\alpha-1} \\
& \times\left\|B\left(u_{f}(s)\right)-B\left(u_{h}(s)\right)\right\| d s \\
\leq & M\left\|z_{1}-z_{2}\right\|_{P C(J, E)}(a+h)+\frac{M\left\|z_{1}-z_{2}\right\|_{P C(J, E)}}{\Gamma(\alpha)} \\
& \times \int_{0}^{t}(t-s)^{\alpha-1} \varsigma(s) d s+\frac{M N}{\Gamma(\alpha)}\left(\int_{0}^{t}(t-s)^{2(\alpha-1)} d s\right)^{1 / 2} \\
& \times\left\|u_{f}-u_{h}\right\|_{L^{2}(J, X)} \\
\leq & M\left\|z_{1}-z_{2}\right\|_{P C(J, E)}(a+h+\delta) \\
& +\frac{M N}{\Gamma(\alpha)}\left\|z_{1}-z_{2}\right\|_{P C(J, E)} N[a+M(a+\delta)] \frac{b^{\alpha-1 / 2}}{\sqrt{2 \alpha-1}} .
\end{aligned}
$$

By interchanging the role of $y_{2}$ and $y_{1}$ in both (94) and (95) we obtain

$$
\left\|\widetilde{R}\left(z_{1}\right)-\widetilde{R}\left(z_{2}\right)\right\| \leq \gamma_{1}\left\|z_{1}-z_{2}\right\|_{P C(J, E)} .
$$

Therefore, $\widetilde{R}$ is contraction, and thus by Theorem 19, $\widetilde{R}$ has a fixed point which is a mild solution for (4).

Next, we give another controllability result.

Theorem 25. Let $\alpha \in(0,1), q \in(0, \alpha), V: L^{2}(J, X) \rightarrow$ $L^{1 / q}(J, E)$ be a bounded linear operator, $A$ is the infinitesimal generator of a strongly semigroup $\{T(t): t \geq 0\}, F: J \times E \rightarrow$ $P_{c k}(E)$ be a multifunction, $g: P C(J, E) \rightarrow E$ and $I_{i}: E \rightarrow$ $E(i=1,2, \ldots, m)$. 
We assume the conditions (HF4), (HF5), (Hg) ${ }^{*}$, and (HI) and the following condition:

$(H \Upsilon)$ the linear bounded operator $\Upsilon: L^{2}(J, X) \rightarrow E$ defined by

$$
\Upsilon(u)=\int_{0}^{b}(b-s)^{\alpha-1} K_{2}(b-s)(V u)(s) d s,
$$

has an invertible $\Upsilon^{-1}: E \rightarrow L^{2}(J, X) / \operatorname{Ker}(W)$, and there exists, a positive constant $L$ such that $\left\|\Upsilon^{-1}\right\| \leq L$ and $\|B\| \leq L$.

Then, (5) is controllable on J provided that

$$
\gamma_{2}=\left[M(a+\delta+h)+\rho L^{2}(a+M a+M \delta)\right]<1 .
$$

Proof. As in the proof of Theorem 21, for every $z \in P C(J, E)$ we define a multivalued $\widetilde{N}: P C(J, E) \rightarrow 2^{P C(J, E)}$ as $y \in R(z)$ if and only if for each $J=1,2, \ldots, m$,

$$
y(t)=\left\{\begin{array}{cc}
K_{1}(t)\left(x_{0}-g(z)\right) \\
\quad+\int_{0}^{t}(t-s)^{\alpha-1} K_{2}(t-s) \\
\times\left(f(s)+\left(V u_{f}\right)(s)\right) d s, \quad t \in J_{0}, \\
K_{1}(t)\left(x_{0}-g(z)\right) \\
\quad+K_{1}\left(t-t_{1}\right) I_{1}\left(z\left(t_{k}^{-}\right)\right) \\
\quad+\int_{0}^{t}(t-s)^{\alpha-1} K_{2}(t-s) \\
\quad \times\left(f(s)+\left(V u_{f}\right)(s)\right) d s, \quad t \in J_{1}, \\
\vdots \quad \\
K_{1}(t)\left(x_{0}-g(z)\right) \\
\quad+\sum_{i=1}^{i=m} K_{1}\left(t-t_{i}\right) I_{k}\left(z\left(t_{k}^{-}\right)\right) \\
\quad+\int_{0}^{t}(t-s)^{\alpha-1} K_{2}(t-s) \\
\quad \times\left(f(s)+\left(V u_{f}\right)(s)\right) d s, \quad t \in J_{m},
\end{array}\right.
$$

where $f$ is an integrable selection for $F(\cdot, z(\cdot))$ and $u_{f}$ is the control function defined in (86). Arguing as in the proof of Theorem 24, we can show that, when using this control any fixed point of $\widetilde{N}$ is the desired mild solution. We will show that $\widetilde{N}$ satisfies the assumptions of Theorem 19 . We only give the main different steps from the proof of Theorem 24. Take $z_{1}, z_{2} \in P C(J, E)$, and let $y_{1} \in \widetilde{R}\left(z_{1}\right)$. Then, there is $f \in$ $S_{F\left(\cdot, z_{1}(\cdot)\right)}^{1}$ such that for any $t \in J_{i}, J=0,1,2, \ldots, m$,

$$
y_{1}(t)=\left\{\begin{aligned}
K_{1}(t)\left(x_{0}-g\left(z_{1}\right)\right) \\
\quad+\int_{0}^{t}(t-s)^{\alpha-1} K_{2}(t-s) \\
\quad \times\left(f(s)+\left(V u_{f}\right)(s)\right) d s, \quad t \in J_{0}, \\
K_{1}(t)\left(x_{0}-g\left(z_{1}\right)\right) \\
\quad+K_{1}\left(t-t_{1}\right) I_{1}\left(z_{1}\left(t_{k}^{-}\right)\right) \\
\quad+\int_{0}^{t}(t-s)^{\alpha-1} K_{2}(t-s) \\
\quad \int_{0}^{t}(t-s)^{\alpha-1} K_{2}(t-s) \\
\quad \times\left(f(s)+\left(V u_{f}\right)(s)\right) d s, \quad t \in J_{1}, \\
\vdots \quad \\
K_{1}(t)\left(x_{0}-g\left(z_{1}\right)\right) \\
\quad+\sum_{k=m}^{k} K_{1}\left(t-t_{k}\right) I_{k}\left(z_{1}\left(t_{k}^{-}\right)\right) \\
\quad+\int_{0}^{t}(t-s)^{\alpha-1} K_{2}(t-s) \\
\quad \times\left(f(s)+\left(V u_{f}\right)(s)\right) d s, \quad t \in J_{m} .
\end{aligned}\right.
$$

As in the proof of Theorem 24 we can find $y_{2} \in \widetilde{N}\left(z_{2}\right)$ such that

$$
y_{2}(t)=\left\{\begin{array}{cc}
K_{1}(t)\left(x_{0}-g\left(z_{2}\right)\right) \\
\quad+\int_{0}^{t}(t-s)^{\alpha-1} K_{2}(t-s) \\
\times\left(h(s)+\left(V u_{h}\right)(s)\right) d s, \quad t \in J_{0}, \\
K_{1}(t)\left(x_{0}-g\left(z_{2}\right)\right) \\
+K_{1}\left(t-t_{1}\right) I_{1}\left(z_{2}\left(t_{k}^{-}\right)\right) \\
\quad+\int_{0}^{t}(t-s)^{\alpha-1} K_{2}(t-s) \\
\quad \times\left(h(s)+\left(V u_{h}\right)(s)\right) d s, \quad t \in J_{1}, \\
\vdots \quad \\
K_{1}(t)\left(x_{0}-g\left(z_{2}\right)\right) \\
\quad+\sum_{k=1}^{k=m} K_{1}\left(t-t_{k}\right) I_{k}\left(z_{2}\left(t_{k}^{-}\right)\right) \\
\quad+\int_{0}^{t}(t-s)^{\alpha-1} K_{2}(t-s) & \\
& \times\left(h(s)+\left(V u_{h}\right)(s)\right) d s, \quad t \in J_{m}
\end{array}\right.
$$

where $h \in S_{F\left(\cdot, z_{2}(\cdot)\right)}^{1}$ and satisfying the relation (90). Arguing as in (93) we get

$$
\left\|u_{f}-u_{h}\right\|_{L^{2}(J, X)} \leq L[a+M a+M \delta]\left\|z_{1}-z_{2}\right\|_{P C(J, E)} .
$$


This inequality with $(\mathrm{Hg})^{*}$ and (98) yields for $t \in J_{0}$

$$
\begin{aligned}
\| y_{1}(t)- & y_{2}(t) \| \\
\leq & M\left\|g\left(z_{1}\right)-g\left(z_{2}\right)\right\|+\frac{M}{\Gamma(\alpha)} \int_{0}^{t}(t-s)^{\alpha-1} \\
& \times\|h(s)-f(s)\| d s+\frac{M}{\Gamma(\alpha)} \int_{0}^{t}(t-s)^{\alpha-1} \\
& \times\left\|\left(V u_{f}\right)(s)-\left(V u_{h}\right)(s)\right\| d s \\
\leq & M a\left\|z_{1}-z_{2}\right\|_{P C(J, E)}+\frac{M}{\Gamma(\alpha)} \int_{0}^{t}(t-s)^{\alpha-1} \varsigma(s) \\
& \times\left\|z_{1}-z_{2}\right\|_{P C(J, E)}+\frac{M}{\Gamma(\alpha)}\left\|V u_{h}-V u_{f}\right\|_{L^{1 / q}\left(J_{0}, E\right)} \\
& \times\left(\int_{0}^{t}(t-s)^{(\alpha-1) /(1-q)} d s\right)^{1-q} \\
\leq & M a\left\|z_{1}-z_{2}\right\|_{P C(J, E)}+M \delta\left\|z_{1}-z_{2}\right\|_{P C(J, E)} \\
& +\rho\|V\|\left\|_{f}-u_{h}\right\|_{L^{2}\left(J_{0}, X\right)} \\
\leq & M a\left\|z_{1}-z_{2}\right\|_{P C(J, E)}+M \delta\left\|z_{1}-z_{2}\right\|_{P C(J, E)} \\
& +\rho L\left\|z_{1}-z_{2}\right\|_{P C(J, E)} L[a+M a+M \delta] \\
& \left\|z_{1}-z_{2}\right\|_{P C(J, E)}\left[M(a+\delta)+\rho L_{P C(J, E)} \cdot(a+M a+M \delta)\right] \\
& \\
& \\
& \\
&
\end{aligned}
$$

Similarly for $t \in J_{i}, i=1,2, \ldots, m$,

$$
\begin{aligned}
\| y_{1}(t) & -y_{2}(t) \| \\
\leq & M\left\|g\left(z_{1}\right)-g\left(z_{2}\right)\right\| \\
& +\sum_{k=1}^{k=m}\left\|K_{1}\left(t-t_{k}\right)\left(I_{k}\left(z_{1}\left(t_{k}^{-}\right)\right)-I_{k}\left(z_{2}\left(t_{k}^{-}\right)\right)\right)\right\| \\
& +\frac{M}{\Gamma(\alpha)} \int_{0}^{t}(t-s)^{\alpha-1}\|h(s)-f(s)\| d s \\
& +\frac{M}{\Gamma(\alpha)} \int_{0}^{t}(t-s)^{\alpha-1}\left\|\left(V u_{f}\right)(s)-\left(V u_{h}\right)(s)\right\| d s \\
\leq & M a\left\|z_{1}-z_{2}\right\|_{P C(J, E)}+M h\left\|z_{1}-z_{2}\right\|_{P C(J, E)} \\
& +\frac{M}{\Gamma(\alpha)} \int_{0}^{t}(t-s)^{\alpha-1} \varsigma(s)\left\|z_{1}-z_{2}\right\|_{P C(J, E)} \\
& +\frac{M}{\Gamma(\alpha)}\left\|V u_{h}-V u_{f}\right\|_{L^{1 / q}\left(J_{0}, E\right)} \\
& \times\left(\int_{0}^{t}(t-s)^{(\alpha-1) /(1-q)} d s\right)^{1-q}
\end{aligned}
$$

$$
\begin{aligned}
\leq & M a\left\|z_{1}-z_{2}\right\|_{P C(J, E)}+M h\left\|z_{1}-z_{2}\right\|_{P C(J, E)} \\
& +M \delta\left\|z_{1}-z_{2}\right\|_{P C(J, E)}+\rho L\left\|z_{1}-z_{2}\right\|_{P C(J, E)} \\
& \times L[a+M a+M \delta] \\
\leq & \gamma_{2}\left\|z_{1}-z_{2}\right\|_{P C(J, E)} .
\end{aligned}
$$

By interchanging the role of $y_{2}$ and $y_{1}$ in both (103) and (104) we get

$$
\left\|\widetilde{R}\left(z_{1}\right)-\widetilde{R}\left(z_{2}\right)\right\| \leq \gamma_{2}\left\|y_{2}-y_{1}\right\|_{P C(J, E)} .
$$

Therefore, $\widetilde{R}$ is contraction, and thus by Theorem $19, \widetilde{R}$ has a fixed point which is a mild solution for the problem (1).

\section{Conclusion}

In this paper, existence and controllability problems of fractional order impulsive semilinear differential inclusions with nonlocal condition have been considered. Some sufficient conditions have been obtained, as pointed in the first section; these conditions are strictly weaker than most of the existing ones. In addition, our technique allows us to discuss some fractional inclusions which contains a linear operator that generates a noncompact semigroup.

\section{Acknowledgments}

The authors thank the referees for their valuable comments and suggestions which improved their paper. This work is supported by the National Natural Science Foundation of China (11201091), Key projects of Science and Technology Research in the Chinese Ministry of Education (211169), Key Support Subject (Applied Mathematics) and Key project on the reforms of teaching contents and course system of Guizhou Normal College.

\section{References}

[1] R. Hilfer, Applications of Fractional Calculus in Physics, World Scientific, Singapore, 1999.

[2] A. A. Kilbas, H. M. Srivastava, and J. J. Trujillo, Theory and Applications of Fractional Differential Equations, vol. 204 of North-Holland Mathematics Studies, Elsevier Science, Amsterdam, The Netherlands, 2006.

[3] K. S. Miller and B. Ross, An Introduction to the Fractional Calculus and Fractional Differential Equations, A Wiley-Interscience, John Wiley \& Sons, New York, NY, USA, 1993.

[4] A. M. A. El-Sayed and A.-G. Ibrahim, "Multivalued fractional differential equations," Applied Mathematics and Computation, vol. 68, no. 1, pp. 15-25, 1995.

[5] B. Ahmad, "Existence results for fractional differential inclusions with separated boundary conditions," Bulletin of the Korean Mathematical Society, vol. 47, no. 4, pp. 805-813, 2010.

[6] R. P. Agarwal, M. Benchohra, and B. A. Slimani, "Existence results for differential equations with fractional order and impulses," Georgian Academy of Sciences A, vol. 44, pp. 1-21, 2008. 
[7] M. Belmekki and M. Benchohra, "Existence results for fractional order semilinear functional differential equations with nondense domain," Nonlinear Analysis. Theory, Methods \& Applications, vol. 72, no. 2, pp. 925-932, 2010.

[8] A. Ouahab, "Fractional semilinear differential inclusions," Computers \& Mathematics with Applications, vol. 64, no. 10, pp. 3235-3252, 2012.

[9] J. Wang, M. Fečkan, and Y. Zhou, "On the new concept of solutions and existence results for impulsive fractional evolution equations," Dynamics of Partial Differential Equations, vol. 8, no. 4, pp. 345-361, 2011.

[10] J. Wang and Y. Zhou, "Existence and controllability results for fractional semilinear differential inclusions," Nonlinear Analysis. Real World Applications, vol. 12, no. 6, pp. 3642-3653, 2011.

[11] Z. Zhang and B. Liu, "Existence of mild solutions for fractional evolutions equations," Journal of Fractional Calculus and Applications, vol. 10, pp. 1-10, 2012.

[12] Y. Zhou and F. Jiao, "Nonlocal Cauchy problem for fractional evolution equations," Nonlinear Analysis. Real World Applications, vol. 11, no. 5, pp. 4465-4475, 2010.

[13] Y. Zhou and F. Jiao, "Nonlocal Cauchy problem for fractional natural evolution equations," Computers \& Mathematics with Applications, vol. 59, pp. 1063-1077, 2010.

[14] M. Benchohra, J. Henderson, and S. Ntouyas, Impulsive Differential Equations and Inclusions, Hindawi Publishing Corporation, Philadelphia, Pa, USA, 2007.

[15] R. A. Al-Omair and A. G. Ibrahim, "Existence of mild solutions of a semilinear evolution differential inclusions with nonlocal conditions," Electronic Journal of Differential Equations, vol. 42, pp. 1-11, 2009.

[16] T. Cardinali and P. Rubbioni, "Impulsive mild solutions for semilinear differential inclusions with nonlocal conditions in Banach spaces," Nonlinear Analysis. Theory, Methods \& Applications, vol. 75, no. 2, pp. 871-879, 2012.

[17] Z. Fan, "Impulsive problems for semilinear differential equations with nonlocal conditions," Nonlinear Analysis. Theory, Methods \& Applications, vol. 72, no. 2, pp. 1104-1109, 2010.

[18] G. M. Mophou, "Existence and uniqueness of mild solutions to impulsive fractional differential equations," Nonlinear Analysis. Theory, Methods \& Applications, vol. 72, no. 3-4, pp. 1604-1615, 2010.

[19] O. K. Jaradat, A. Al-Omari, and S. Momani, "Existence of the mild solution for fractional semilinear initial value problems," Nonlinear Analysis. Theory, Methods \& Applications, vol. 69, no. 9, pp. 3153-3159, 2008.

[20] E. A. Dads, M. Benchohra, and S. Hamani, "Impulsive fractional differential inclusions involving the Caputo fractional derivative," Fractional Calculus \& Applied Analysis, vol. 12, no. 1, pp. 15-38, 2009.

[21] J. Henderson and A. Ouahab, "Impulsive differential inclusions with fractional order," Computers \& Mathematics with Applications, vol. 59, no. 3, pp. 1191-1226, 2010.

[22] A. G. Ibrahim and N. A. Al Sarori, "Mild solutions for nonlocal impulsive fractional semilin ear differential inclusions with delay in Banach spaces," Applied Mathematics, vol. 4, pp. 40-56, 2013.

[23] E. Hernández M. and D. O’Regan, "Controllability of VolterraFredholm type systems in Banach spaces," Journal of the Franklin Institute. Engineering and Applied Mathematics, vol. 346, no. 2, pp. 95-101, 2009.
[24] V. Obukhovski and P. Zecca, "Controllability for systems governed by semilinear differential inclusions in a Banach space with a noncompact semigroup," Nonlinear Analysis. Theory, Methods \& Applications, vol. 70, no. 9, pp. 3424-3436, 2009.

[25] M. Guo, X. Xue, and R. Li, "Controllability of impulsive evolution inclusions with nonlocal conditions," Journal of Optimization Theory and Applications, vol. 120, no. 2, pp. 355-374, 2004.

[26] M. Benchohra, J. Henderson, S. K. Ntouyas, and A. Ouahab, "Existence results for fractional functional differential inclusions with infinite delay and applications to control theory," Fractional Calculus \& Applied Analysis, vol. 11, no. 1, pp. 35-56, 2008.

[27] N. K. Tomar and J. Dabas, "Controllability of impulsive fractional order semilinear evolution equations with nonlocal conditions," Journal Nonlinear Equations and Applications, pp. 57-67, 2012.

[28] J. Wang and Y. Zhou, "A class of fractional evolution equations and optimal controls," Nonlinear Analysis. Real World Applications, vol. 12, no. 1, pp. 262-272, 2011.

[29] J.-P. Aubin and H. Frankowska, Set-Valued Analysis, vol. 2, Birkhäuser, Boston, Ma, USA, 1990.

[30] H. Covitz and S. B. Nadler, "Multi-valued contraction mappings in generalized metric spaces," Israel Journal of Mathematics, vol. 8, pp. 5-11, 1970.

[31] M. Kamenskii, V. Obukhowskii, and P. Zecca, Condensing Multivalued Maps and Semilin-Ear Differential Inclusions in Banach Spaces, vol. 7 of De Gruyter Series in Nonlinear Analysis and Applications, Walter, Berlin, Germany, 2001.

[32] C. Castaing and M. Valadier, Convex Analysis and Measurable Multifunctions, vol. 580 of Lecture Notes in Mathematics, Springer, Berlin, Germany, 1977.

[33] S. Hu and N. S. Papageorgiou, Handbook of Multivalued Analysis. Vol.I, vol. 419 of Theory in Mathematics and Its Applications, Kluwer Academic, Dordrecht, The Netherlands, 1997.

[34] S. Hu and N. S. Papageorgiou, Handbook of Multivalued Analysis. Vol. II, vol. 500 of Theory in Mathematics and Its Applications, Kluwer Academic, Dordrecht, The Netherlands, 2000.

[35] H.-P. Heinz, "On the behaviour of measures of noncompactness with respect to differentiation and integration of vector-valued functions," Nonlinear Analysis. Theory, Methods \& Applications, vol. 7, no. 12, pp. 1351-1371, 1983.

[36] D. Bothe, "Multivalued perturbations of $m$-accretive differential inclusions," Israel Journal of Mathematics, vol. 108, pp. 109-138, 1998.

[37] R. Bader, M. Kamenskiǔ, and V. Obukhovskiŭ, "On some classes of operator inclusions with lower semicontinuous nonlinearities," Topological Methods in Nonlinear Analysis, vol. 17, no. 1, pp. 143-156, 2001.

[38] X.-B. Shu, Y. Lai, and Y. Chen, "The existence of mild solutions for impulsive fractional partial differential equations," Nonlinear Analysis. Theory, Methods \& Applications, vol. 74, no. 5, pp. 2003-2011, 2011.

[39] M. Fečkan, Y. Zhou, and J. Wang, "On the concept and existence of solution for impulsive fractional differential equations," Communications in Nonlinear Science and Numerical Simulation, vol. 17, no. 7, pp. 3050-3060, 2012. 


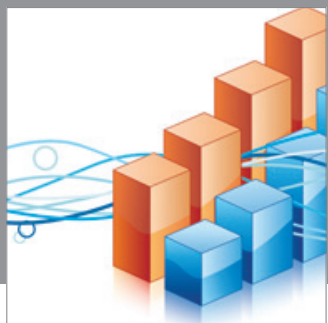

Advances in

Operations Research

mansans

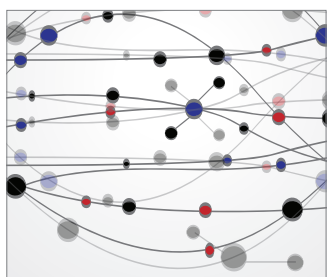

The Scientific World Journal
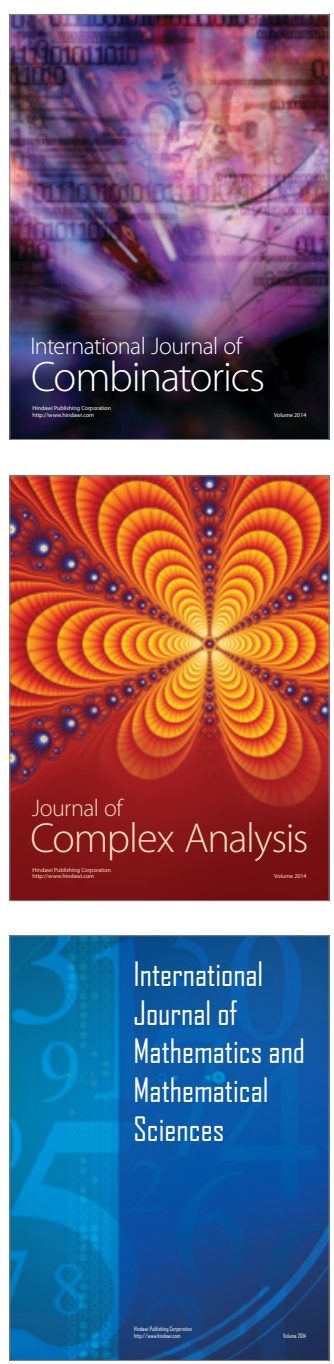
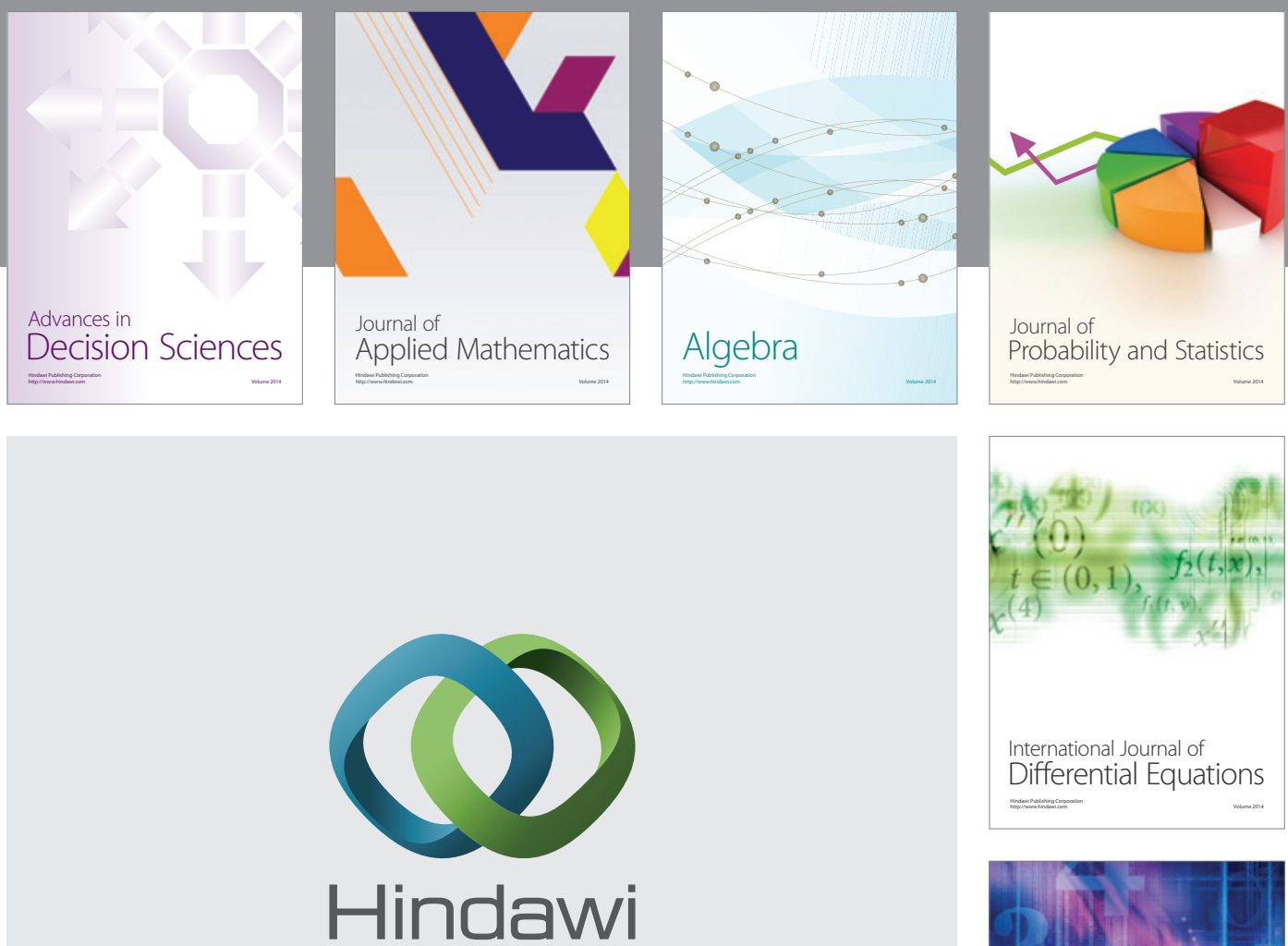

Submit your manuscripts at http://www.hindawi.com
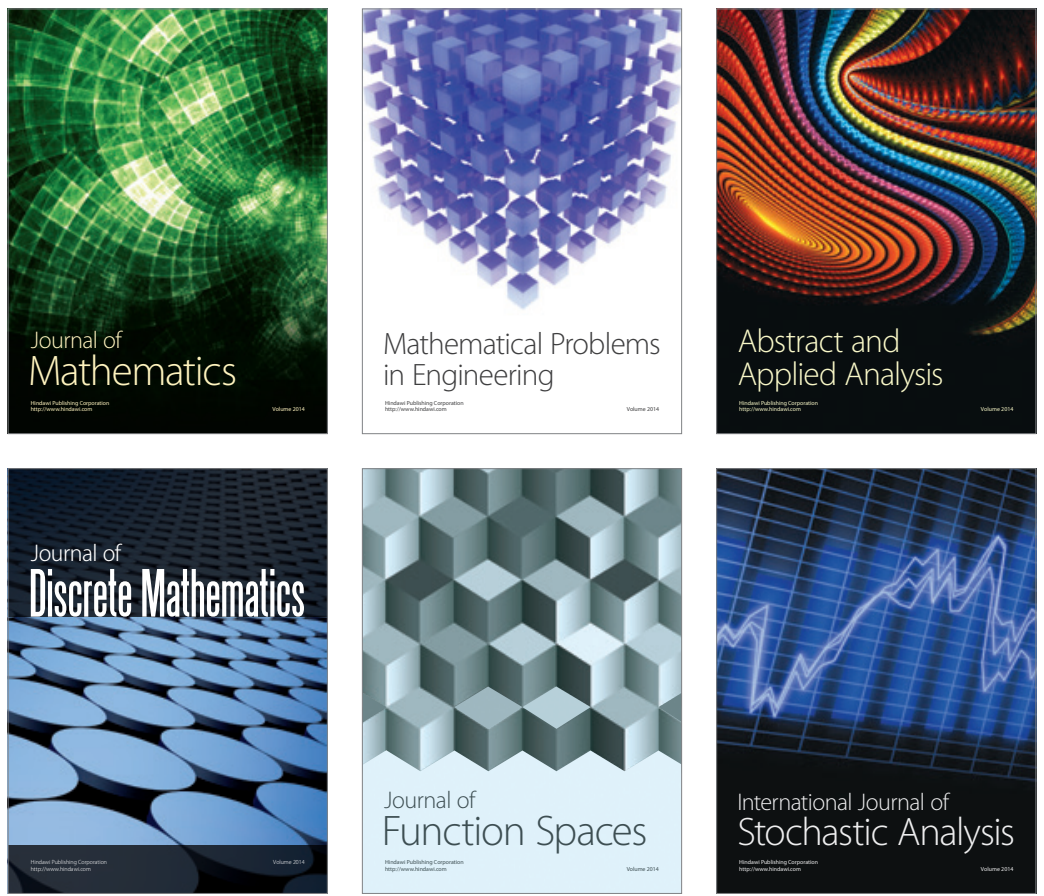

Journal of

Function Spaces

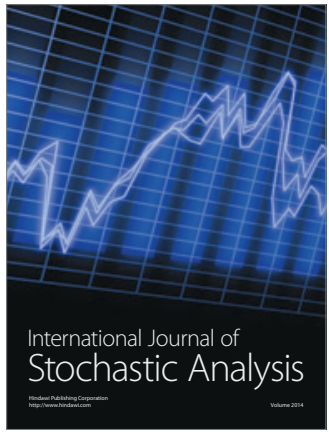

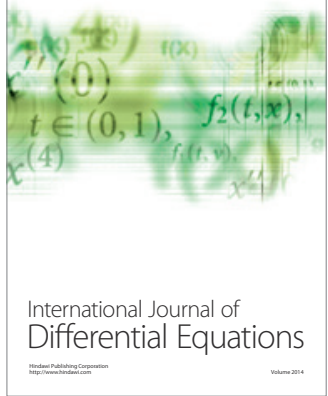
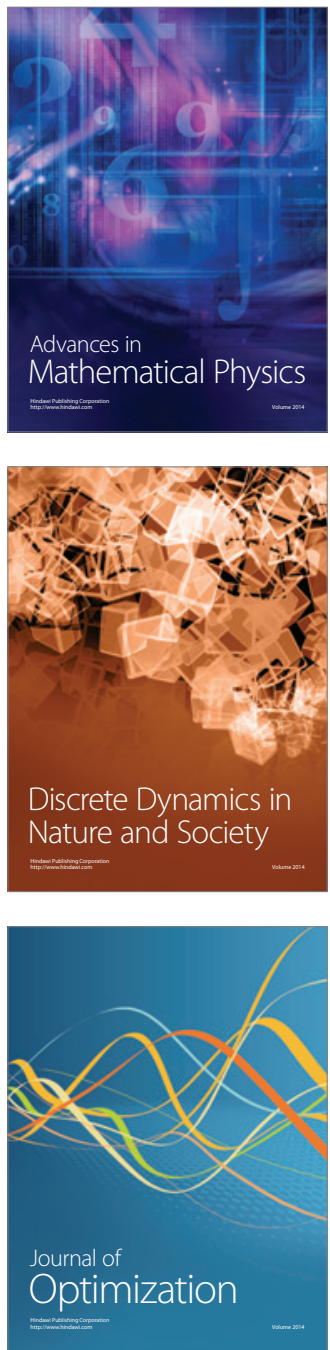